\title{
Shifting qualities of negative affective experience through adolescence: Age-related change and associations with functional outcomes
}

\author{
Katherine A. Grisanzio, John C. Flournoy, Patrick Mair, Leah H. Somerville \\ Department of Psychology and Center for Brain Science, Harvard University
}

Abstract word count: $\quad 255$

Body word count: $\quad 6,701$

Acknowledgments: The authors thank Graham Baum, Brenden Tervo-Clemmens, and Shirley Wang for guidance on data analysis and data presentation. The authors also thank Deanna Barch, Melanie Grad-Freilich, Cynthia Hodge, Kat Kabotyanski, and Petra Lenzini for assistance with data acquisition protocols and data access. Finally, the authors would like to acknowledge the efforts of the Human Connectome Project in Development Consortium (PIs: Deanna Barch, Susan Bookheimer, Randy Buckner, Mirella Dapretto, Stephen Smith, Leah Somerville, Kathleen Thomas, David Van Essen, and Essa Yacoub).

\section{Funding:}

Research reported in this publication was supported by NIH grants U01MH109589 and U01MH109589-S1 and by the 14 NIH Institutes and Centers that support the NIH Blueprint for Neuroscience Research, by the McDonnell Center for Systems Neuroscience at Washington University, and by the Office of the Provost at Washington University. This work was also supported by a National Science Foundation Graduate Research Fellowship (DGE1745303) to KAG.

Conflict of Interest Statement: The authors declare no conflicts of interest.

The processed data and analysis code can be found at https://osf.io/dsxnu/. 


\begin{abstract}
Research shows negative affect increases in healthy adolescents, and this normative change is paralleled by increasing risk for the onset of psychopathology. However, research is limited in characterizing qualitative differences in the type of negative affect experienced beyond the positive-negative valence dimension. In the current study, we establish the relationship between different forms of negative affect and functioning outcomes (i.e., different facets of social functioning and life satisfaction), and examine whether these forms of negative affect are differentially prevalent across late childhood and adolescence. 770 participants aged 8-17 years completed self-report measures that assessed a wide range of negative affective experiences. A factor analysis on the negative affect items revealed a 4-factor solution that characterized the dimensions of affective experience, with factors reflecting general anxiety, anger, evaluative anxiety, and sadness. Generalized additive model approaches revealed general anxiety increased non-linearly with age and was associated with decreased reports of emotional support, a facet of social functioning. Anger was associated with increased perceived hostility, perceived rejection, and decreased life satisfaction, and remained stable across the age range. Evaluative anxiety was associated with greater loneliness and increased linearly with increasing age. Sadness was associated with all outcome measures and showed non-linear changes with age, with notable increases in mid-adolescence. These results show that subsuming these subtypes of negative affect under a singular concept may obscure meaningful relationships between affect, age, and functioning. Exploring diverse forms of negative affect may help refine theories of emotional development and ultimately inform windows of risk for psychopathology.
\end{abstract} Keywords: Emotion, affect, development, childhood, adolescence 


\section{Introduction}

Adolescence is a developmental transition beginning around the onset of puberty and ending when a person reaches adult-like levels of independence, and this transition is marked by substantial change at the experiential and psychological levels (Somerville, 2016). Research shows that among healthy adolescents, there is a downward shift in emotional experience in the direction of more frequent negative and less frequent positive states (Larson et al., 2002). While this increase in negative emotional experience, or negative affect, is thought to be developmentally normative, current theories suggest that experiences and regulation of negative emotion can contribute to the onset and maintenance of adolescent psychopathology. For example, increases in the intensity, frequency, and lability of negative affect and difficulties in emotion regulation have been linked to depression symptoms in adolescence (Silk et al., 2003;

Larson et al., 1990). More broadly, adolescence is characterized by increased risk for the onset of many forms of psychopathology, such as major depression, eating disorders, and substance use disorders (Kessler et al., 2005). While many cases of psychopathology remit before adulthood, research shows that about half of boys and two-thirds of girls with psychopathology during adolescence will go on to experience at least one further episode in young adulthood (Patton et al., 2014). Thus, adolescence is a key phase of life in which emotional experiences transform and may confer tangible, long-term health risks.

Much of the previous research on affective experience during adolescence has measured affect based on a positive-negative valence dimension (e.g., Larson et al., 2002; Larson \& Richards, 2000) or using single-item measures of affect (e.g., the semantic differential SadHappy scale with "sad" and "happy" on polar ends; Moneta et al., 2001). However, within the broad category of negative valence, individuals frequently experience affect as a more precise 
feeling state (e.g., anger, fear, sadness) (Russell, 1980). Previous research examining negative affect as a singular concept has been limited in its capacity to characterize the range of negative affective experiences and their expression over development.

Research suggests there is value in examining affect, and specifically affectpsychopathology relationships, at this more granular level. Watson and colleagues (2011) found that in adults, general negative affect showed broad and nonspecific associations with internalizing psychopathology, but when different forms of negative affect were examined at a more detailed level, specific relationships emerged. For example, the extent to which participants reported feeling fear mimicked general negative affect as a broad predictor of psychopathology, whereas feelings of sadness and guilt were related to major depression but had weaker links to anxiety disorders, and anger and hostility displayed relatively weak associations with the disorders examined. This research suggests that different forms of negative affect have distinct correlates, highlighting the limitations of previous studies that have relied on global positive and negative affect scores.

Because little work has been done to comprehensively characterize different forms of negative affect in adolescence, we know little about the relationship between qualitatively different forms of negative affect and daily functioning, such as social functioning, in youth. A critical domain of change in an adolescent's life is in the social realm, as they begin to spend more time with peers and are increasingly confronted with social evaluation (Rudolph \& Hammen, 1999; Rudolph et al., 2000). While research shows close links exist between social and affective experiences during this adolescent period (e.g., Rodman et al., 2017, Somerville, 2013), few studies have examined the relationship between different forms of negative affect and social functioning. 
More globally, negative affect is also associated with lower life satisfaction, or a cognitive evaluation of one's life as a whole. Negative affect consistently predicts lower satisfaction with life across numerous studies (e.g., Pilcher 1998; Thompson et al. 2007) and is inversely associated with life satisfaction across the adult lifespan (Siedlecki et al. 2008). Satisfaction with life is an important part of psychological health and well-being (Diener, 1984; Pavot \& Diener, 2008) and predicts many physical health outcomes, such as increased mortality (e.g., Chida \& Steptoe, 2008). The current study explores this association between negative affect and life satisfaction at a more detailed level by identifying the relationships between different forms of negative affect and life satisfaction in a large youth sample.

In addition to a focus on the valence dimension, previous work has been limited by small participant samples (e.g., Larson et al., 2002), with some exceptions (e.g., Moneta et al., 2001). To address limitations outlined above in the prior work, in the current study we identify and characterize negative affective experiences in a subset of children and adolescents using selfreport data from the Human Connectome Project in Development (Somerville et al., 2018), a large-scale study of brain connectivity and psychological development in youth. Moreover, efforts to recruit a representative sample with respect to race, ethnicity, sex, and socioeconomic status ensures a high degree of generalizability to youth in the United States. In the current study, we 1) apply a data-driven approach to identify the latent variables represented within negative affect items, 2) identify the degree to which different forms of negative affect are associated with social functioning, 3) identify the degree to which forms of negative affect are associated with global life satisfaction, and 4) identify and describe age-related changes in the forms of negative affect across the sample, aged 8-17 years. While our primary focus is on adolescence, we examined children as young as 8 to inform the childhood to adolescent transition; thus, our 
sample is well suited to characterize experiences from late childhood to adolescence. While prior research informs the expected negative relationship between negative affect as a whole and the functioning measures, the relationship between different forms of negative affect and social functioning and life satisfaction were considered exploratory. Similarly, we predicted global negative affect to increase with increasing age, but tests for distinct age-related patterns for different forms of negative affect were considered exploratory.

\section{Methods}

\section{Participants}

The current study examined data from a subset of participants from the Human Connectome Project in Development (HCP-D; Somerville et al., 2018). We report how we determined our sample size, all data exclusions, all manipulations, and all measures in the study. Data from seven hundred and eighty-three participants ages eight to seventeen years were made available at the time of analysis from the HCP-D investigators in advance of public data release. Participants ages 5-7 years were not included because the only affect measures available were parent-report (not self-report), and participants 18 years and older were not included in the current analysis due to a lack of overlap in measures.

Data from thirteen participants were excluded due to incomplete data (i.e., greater than $20 \%$ of affect data missing; see Supplementary Section 1 for flow chart of sample selection). Remaining missing data was median replaced. For the life satisfaction analysis, 544 participants (ages $8.00-17.97$ years, mean age $=11.53$ years) with overlapping data were analyzed, as the remaining participants completed a different subset of life satisfaction items. 
Thus, final analysis included data from 770 participants (see Supplementary Section 2 for age distribution; age range $=8.01-17.97$ years, $M_{\mathrm{age}}=12.60, S D_{\mathrm{age}}=2.84,49.6 \%$ female, 15.7\% Hispanic or Latino, 70.4\% White, 15.5\% Black/African American, 1.4\% Native American, 0.4\% Native Hawaiian or other Pacific Islander, 8.8\% Asian, 18.8\% more than one race, $1.6 \%$ don't know). Gender was defined based on parent-reported gender identity reported during the phone screening, and no participants were excluded from analyses of gender differences. Sampling aimed to match the diversity across ethnicity, race, and socioeconomic status in the United States (Somerville et al., 2018). All participants were fluent in English and did not have cognitive impairments limiting their ability to provide assent or complete tasks. To preserve heterogeneity in the sample, participants were included if they had elevated symptoms of psychiatric illness as long as they had not been diagnosed and treated for 12 months or longer (see Supplementary Section 3 for overview of inclusion and exclusion criteria).

Participants were recruited from four sites across the USA: Harvard University, University of California - Los Angeles (UCLA), University of Minnesota (UMinn), and Washington University in St. Louis (WUSTL) (see Supplementary Section 4 for participant demographics by site). Guardians of minor participants provided informed consent prior to participation, and minors assented to participation. All procedures were approved by a central Institutional Review Board administered at Washington University in St. Louis.

\section{Measures}

The current study focuses on a subset of measures from the broader HCP-D project that were selected a priori to match the research questions described above. We selected measures with items assessing negative affective experience (i.e., subjective experiences of one's 
emotional state), social functioning, and life satisfaction for analysis, as described below. We then applied a data-driven approach to identify the latent variables represented within these items.

All measures analyzed were self-reported by the participant. To ensure comprehension and accuracy in reporting for younger participants (ages 8 to 11 years), the experimenter read the survey items out loud and watched as the child made their selection. Many items used in analysis were drawn from the NIH Toolbox (Gershon et al., 2013), a set of age-specific measurements that were developed and validated to be psychometrically sound in each of the age groups surveyed. All NIH Emotion Toolbox measures are described below using descriptions from the NIH Toolbox Scoring and Interpretation Guide (https://nihtoolbox.my.salesforce.com/sfc/p/\#2E000001H4ee/a/2E0000004yR3/Ckb_AKw1oFU C56tgf6tdxcGDYaYbu8rsmBSFOX2Ec4g) and the NIH Toolbox Brochure (http://www.healthmeasures.net/ images/nihtoolbox/NIH_Toolbox_brochure_June_2017.pdf)

\section{Negative Affect Experience}

The NIH Emotion Toolbox negative affect measures assess the emotional domains of sadness, fear, and anger by asking participants to rate how often they have felt specific emotional experiences within the past seven days on a 5-point Likert scale from "Never" to "Almost Always". The Sadness Survey measures unpleasant feelings or emotions of sadness; the Fear Survey assesses fear, anxious misery, and hyperarousal; and the Anger Survey assesses angry mood and aggression (verbal and physical). We also used a child-adapted version of the behavioral inhibition scale from the Behavioral Inhibition/Behavioral Activation Scale 
(BIS/BAS; Pagliaccio et al., 2016; Carver \& White, 1994), which measures concern about possible negative or punishing events and sensitivity to the occurrence of such events. Participants rated statements about their thoughts and feelings on a 4-point Likert scale from "Not True" to "Very True". All items were administered on digital platforms, as described in Somerville et al. (2018).

From these measures, we selected items for analysis using two criteria. First, as we are interested in characterizing affect, we selected items that specifically assessed affective experiences (i.e., the subjective experience of valence and arousal), rather than emotion-related thoughts or beliefs (e.g., "I felt upset" describes an affective state and was included, whereas "I felt everything in my life went wrong" describes a belief and was excluded). Second, we included items that were administered to, and completed by participants, in the entire age range. For the NIH Emotion Toolbox modules, participants of different ages received a different number of items, and some modules were Computer Adaptive Tests (i.e., the test varied based on the participants' answers). Therefore, if an item was only administered to a subset of participants in the 8-17 year age range, the item was excluded from analysis. See Supplementary Section 5 for the final selected items.

\section{Social Functioning}

The NIH Emotion Toolbox modules measuring the structure, extent, and quality of social relationships were used to measure social functioning. These modules include the Emotional Support Survey (measuring the perception that people in one's social network are available to listen to one's problems with empathy, caring, and understanding), the Friendship Survey (measuring the perceptions of the availability of friends or companions with whom to interact or 
affiliate), the Perceived Hostility Survey (self-reported perceptions of hostility, e.g., measuring the perceptions of how often people argue with me, yell at me, or criticize me), the Perceived Rejection Survey (measuring perceived insensitivity, e.g., how often people don't listen when I ask for help, or don't pay attention to me), and the Loneliness Survey (measuring the perception that one is alone, lonely, or socially isolated from others). For the Emotional Support and Friendship surveys, higher scores indicate better social functioning (i.e., increased emotional support and friendship); for the Perceived Hostility, Perceived Rejection, and Loneliness surveys, higher scores indicate poorer social functioning (i.e., increased perceived hostility, perceived rejection, and loneliness).

Items that query the juncture of social and affective experiences (e.g., experiences of loneliness) required a decision to categorize the item as "affective" or "social" for analyses. In these cases, we followed the original categorization made during the construction of the NIH Toolbox categories. Thus, experiences of loneliness were characterized as reflecting the social domain.

\section{Life Satisfaction}

To measure life satisfaction, the NIH Emotion Toolbox General Life Satisfaction Survey was used. This survey measures global feelings and attitudes about one's life (e.g., my life is going well). 


\section{Aims and Analyses}

Exploratory Factor Analysis

The first aim of the study was to uncover the latent structure of the negative affect variables to obtain meaningful summary scores for different forms of negative affect in our sample. We implemented a data-driven approach to identify forms of negative affect rather than relying on scale summary scores, as this approach 1) allowed us to only include items measuring negative affective experience, rather than emotion-related thoughts or beliefs, and 2) allowed items capturing similar affective experiences to group together without being tied to a priori assumptions.

To achieve aim, we conducted an exploratory factor analysis (EFA) using the $f a$ function from the psych package (version 1.8.12, Revelle, 2018) in R (R Core Team, 2020). Twenty-two items measuring a range of negative affective experiences were selected from the self-report measures. Two items were eliminated due to consistently low loadings across bootstrapped samples in a supplementary analysis (see Supplementary Section 6) and the remaining twenty items were input to the EFA. Because the items all measure negative affect and are assumed to be related, we used an oblimin rotation to achieve a non-orthogonal (oblique) solution that would allow the factors to be correlated. Additionally, the negative affect variables were all 4 or 5-point Likert scale items, and thus polychoric correlations were used in the EFA to accurately estimate the correlations between the ordinal variables. When choosing the number of factors to retain, we considered a scree plot using the elbow method, eigenvalues $>1$ criteria, parallel analysis (in which a factor is considered as "significant" if its eigenvalue is larger than the $95 \%$ quantile of those obtained from a random data matrix of the same size as the original), and interpretability. Because parallel analysis is cautioned to be partially sensitive to sample size (i.e., for large 
samples, the eigenvalues of random factors will tend to be very small resulting a larger number of factors than using other criteria; Revelle (2015)), the parallel analysis was weighted less strongly. The resulting factor solution was compared to three additional theoretically identified and data-driven factor solutions to ensure it was the best fitting model (see Supplementary Section 7).

While our primary analyses focus on forms of negative affect, to compare our results with prior work that has studied negative affect as a singular concept, we conducted a supplementary analysis of overall negative affect, in which all negative affect measures were combined into a single score using factor analysis (see Supplementary Section 8).

\section{Confirmatory Factor Analysis}

To evaluate the fit of the factor structure extracted in the EFA, we conducted a confirmatory factor analysis (CFA) using the $c f a$ function in R's lavaan package (version 0.6-9, Rosseel, 2012). We calculated and report the standard measures and fit rules (Hu \& Bentler, 1999) to assess how well the proposed model produced by the EFA captures the covariance between the measured items. The fit indices used include the comparative fit index (CFI, should be $>=0.95$ ), root mean square error of approximation (RMSEA should be $<=0.05$, upper CI bound $<=0.10$ ), standardized root mean square residual (SRMR, should be $<=0.08$ ), TuckerLewis index (TLI, > .90 indicates good fit), McDonald fit index (MFI, higher values indicate better fit), and the chi-squared test (a non-significant p-value suggests the model fits) was also used. While we report the chi-square for completeness, this statistic becomes more significant with larger samples and has low power at smaller samples (Gatignon, 2010); due to the large sample size in the current study, we did not rely on this statistic for determining fit. To improve 
model fit, we identified and removed items with non-significant loadings. These fit indices represent an upper bound on the fit that would be expected in an independent sample because the model was developed and tested using the same data.

\section{Relationship between Negative Affect and Social Functioning}

The second aim of the study was to identify the degree to which different facets of negative affect were significantly associated with social functioning. Twenty-nine social functioning items were taken from five different modules in the NIH Emotion Toolbox measuring emotional support, friendship, perceived hostility, loneliness, and perceived rejection. We calculated summary scores by summing the Likert response scores for each social functioning domain ("Never" =1, "Rarely" =2, "Sometimes" =3, "Usually" = 4, "Always" = 5). We chose to undertake the factor analysis approach for modeling the latent structure of negative affect items, as it allowed us to select only questions about affect, did not require $a$ priori assumptions about how items would group together. By contrast, the social functioning outcome items were all taken directly from existing sub-scales from the NIH Emotion Toolbox modules as they more straightforwardly represented the constructs of interest.

To test the extent to which the negative affect factors relate to the identified social domains across the age range as a whole, we fit regression models in $\mathrm{R}$ using the $m g c v$ package (version 1.8-28, Wood, 2003). For each social domain, a separate regression model was run to identify which negative affect factors (all included as linear predictors within the same model) are significantly associated with that social functioning domain score. Thus, the parameter estimates were conditioned on the other negative affect variables in the model. Development leads to linear and non-linear changes in both affect and social functioning, so the spline of age 
was included as a control. Standardized regression coefficients $(\beta)$ are reported, which can be interpreted as effect sizes (such that one standard deviation of the predictor results in $\beta$ standard deviations change in the outcome). Multiple comparisons were accounted for using the Holm method, which controls the family-wise error rate (Holm, 1979).

We additionally evaluated whether the negative affect factors had differential effects on the outcome variable (compared to a null hypothesis where all factors have an equal impact). We fit two regression models: one in which the coefficients were allowed to differ for each of the negative affect factors, and one in which the coefficients were constrained to be equal across all affect factors. The two models were then compared using Akaike Information Criterion (AIC) values, and we selected the one with the smallest value. The difference in AIC values between the two models ( $\triangle \mathrm{AIC}$ ) is reported.

\section{Relationship between Negative Affect and Life Satisfaction}

The third aim was to identify the degree to which different forms of negative affect relate to overall life satisfaction. We calculated a summary score for life satisfaction by summing the Likert response scores from the items in the General Life Satisfaction survey from the NIH Toolbox. To test the extent to which the negative affect factors are associated with life satisfaction, we conducted linear regression models as described above. Life satisfaction was the dependent variable and all negative affect factors (in addition to the spline of age) were included in the same model as independent variables. 
Age-Related Changes in Negative Affect

The final aim of the study was to identify and describe age-related changes in the negative affect factors across our sample, aged 8-17 years. Because age trends are not limited to linear patterns, we compared models with age modeled both linearly and non-linearly.

To explore non-linear age-related changes, we used generalized additive models (GAM) with thin-plate regression splines (see Wood, 2017). This method produces regression equations that fit the data using cross-validation procedures but are also penalized for the number of parameters to prevent overfitting (Wood, 2003). The result of these models is a stable, smooth curve that describes the data's age-related change but is not constrained to stereotyped linear, quadratic, or cubic shapes.

For each negative affect factor, we tested a linear and spline model, with the negative affect factor as the dependent variable and age as the independent variable, as well as a null model with no age predictors. Linear analyses were tested using the $l m$ function in R's stats package (R Core Team, 2020), and GAM models were tested using the $g a m$ function in the $m g c v$ package. The two models were then compared using AIC values. The model with the lowest AIC was considered the best fit and interpreted. In cases where the spline model yielded a linear fit, the AIC values for the linear and spline model are identical and a linear model should be interpreted. Multiple comparisons were accounted for using the Holm method.

To fully describe the age-related changes in the negative affect factors, we aimed to identify the specific windows of age during which experiences of that form of negative affect significantly increased or decreased. Unlike linear models, which are restricted to identifying a constant rate of change, spline models may identify age-related increases or decreases in some parts of the curve and not others. Thus, for negative affect factors for which the spline model was 
the best fitting model, post-hoc analyses identified the windows of accelerated change across the age range. We calculated the first derivative of the smooth function of age using finite differences (a method used to approximate derivatives where the derivative is calculated at a set number of points), and a simultaneous $95 \%$ confidence interval for 100 points along the derivative (Ruppert et al., 2003). The set of simultaneous confidence intervals is defined so that, given other modeling assumptions, the true population value (e.g., a negative affect factor at a certain age) falls outside the set at the nominal rate $(5 \%$, in this case). This ensures that inferences based on our set of 100 confidence intervals are adjusted for multiple comparisons. Intervals of change can be identified as areas where the simultaneous confidence interval of the derivative does not include zero. The derivatives function in R's gratia package (version 0.4.1, Simpson, 2020) was used to calculate the derivatives and simultaneous confidence intervals (Simpson, 2020). The processed data ${ }^{1}$ and analysis code can be found at https://osf.io/dsxnu/. This study was not preregistered.

Although outside of the scope of the primary line of questioning, we conducted additional analyses 1) testing the robustness of the factor solution across age and gender, 2), testing gender as a moderator in affect - functioning relationships, 3) evaluating the relations between pubertal stage and negative affect, 4) characterizing the relationship between age and social functioning and life satisfaction measures, and 5) evaluating the relationship between the primary measures and a dimensional self-report measure of psychopathology. See the Supplementary Materials for the methods and results of these additional inquiries.

\footnotetext{
${ }^{1}$ Due to HCP-D sharing restrictions we are not able to post the full data, but permission to access the raw data may be requested at https://www.humanconnectome.org/study/hcp-lifespan-development/data-releases
} 


\section{Results}

\section{Exploratory Factor Analysis}

The scree plot (see Supplementary Section 9) indicated that 2 or 5 factors may be optimal, the eigenvalues $>1$ criteria indicated 3 factors, and the parallel analysis indicated 5 factors. Two, 3, 4, 5-factor solutions were extracted to compare interpretability. A 2-factor solution (52.3\% of variance explained) consisted of a factor with items relating to evaluative anxiety (i.e., items centered around anxiety about making mistakes or being negatively evaluated) and a factor with anger, general anxiety (i.e., general feelings of worry, fear, or nervousness), and sadness items. A 3-factor solution (59.6\% of variance explained) consisted of a general anxiety factor, an anger factor, and an evaluative anxiety factor, with sadness items loading weakly on the anxiety and anger factors. A 4-factor solution (64.5\% of variance explained) resulted in a general anxiety factor, an anger factor, an evaluative anxiety factor, and a sadness factor. A 5-factor solution (68.5\% of variance explained) was consistent with the 4factor solution with an added factor of one item, "I usually get very tense when I think something unpleasant is going to happen". Due to common conceptualization of anger, anxiety, and sadness as distinctly experienced negative emotions (Russell, 1980) we eliminated the 2-factor solution. Due to the weak loadings of the sadness items, we eliminated the 3-factor solution. Finally, due to the difficulty of interpreting the $5^{\text {th }}$ factor distinctly from the general anxiety and evaluative anxiety factors of the 4-factor solution and the limited utility in a 1-item factor, we chose the 4factor solution as the optimal solution (Figure 1).

Due to the data-driven nature of our approach, we selected the following names to describe the affective state expressed in each group of items without direct reference to the original scales or to clinical terminology: general anxiety, anger, evaluative anxiety, and 
sadness. General anxiety contained items originally from the NIH Toolbox Fear Survey, anger contained items from the NIH Toolbox Anger Survey, sadness contained items from the NIH Toolbox Sadness and Anger Surveys, and evaluative anxiety contained items originally from the BIS scale.

The correlations between factors were as follows: general anxiety and anger $(r(768)=$ $.61, p<.001)$, general anxiety and evaluative anxiety $(r(768)=.31, p<.001)$, general anxiety and sadness $(r(768)=.64, p<.001)$, anger and evaluative anxiety $(r(768)=.30, p<.001)$, anger and sadness $(r(768)=.45, p<.001)$, and evaluative anxiety and sadness $(r(768)=.18, p<.001)$.

\section{Confirmatory Factor Analysis}

The CFA indicated that all items had significant loadings (range of standardized loadings: $.623-.877$ ), so all items were retained for the analysis (see Supplementary Section 10 for all parameter estimates). The fit statistics of the final four-factor solution were as follows: chisquared test statistic $=523.26(\mathrm{df}=164, p<0.001), \mathrm{CFI}=.992, \mathrm{RMSEA}=.053(\mathrm{CI}=.048$, $.059), \mathrm{SRMR}=.051, \mathrm{TLI}=.991, \mathrm{MFI}=.792$. The CFI, SRMR, and TLI are all within the recommended range to suggest a well-fitting model $(\mathrm{CFI}>=.95$, SRMR $<=.08$, TLI $>.90)$. The RMSEA is slightly higher than the recommended value $(<=.05)$, however, the upper CI bound is within the recommended range $(<=.10)$. Therefore, taken together, the CFA fit indices suggest the four-factor solution is a well-fitting model.

\section{Relationship between Negative Affect and Social Functioning}

Table 1 presents the results of the linear models conducted to identify which negative affect factors were significantly associated with different domains of social functioning. For all 
significant relationships, increased negative affect was associated with decreased social functioning. General anxiety was significantly associated with lower reports of emotional support $(\beta=-.16, p=.024)$. Anger was significantly associated with greater perceived hostility $(\beta=.34, p<.001)$ and perceived rejection $(\beta=.23, p<.001)$. Evaluative anxiety was significantly associated with greater loneliness $(\beta=.14, p<.001)$. Sadness was significantly associated with lower reports of emotional support $(\beta=-.16, p=.007)$ and friendship $(\beta=-.21, p$ $<.001)$, and greater perceived hostility $(\beta=.15, p=.003)$, loneliness $(\beta=.47, p<.001)$, and perceived rejection $(\beta=.28, p<.001)$. The unconstrained models (where the coefficients were allowed to differ for each of the negative affect factors) had a lower AIC value than the constrained models (where the coefficients were equal for all negative affect factors) for all regressions: emotional support $(\Delta \mathrm{AIC}=17.07)$, friendship $(\triangle \mathrm{AIC}=4.64)$, perceived hostility $(\Delta \mathrm{AIC}=17.28)$, loneliness $(\Delta \mathrm{AIC}=61.17)$, and perceived rejection $(\Delta \mathrm{AIC}=11.71)$.

The spline of age in the above models was significant in the emotional support $(F=5.55$, $p<.001)$ and perceived hostility $(F=16.24, p<.001)$, and was not significant in friendship $(F=$ $1.78, p=.124)$, loneliness $(F=1.45, p=.230)$, or perceived rejection $(F=1.20, p=.322)$. For the significant age terms, increasing age is related to higher levels of emotional support and lower levels of perceived hostility.

\section{Relationship between Negative Affect and Life Satisfaction}

The results of the linear model to identify which negative affect factors were significantly associated with life satisfaction are summarized in Table 1. Sadness $(\beta=-.28, p<.001)$ and anger $(\beta=-.22, p<.001)$ were significantly associated with decreased life satisfaction. The unconstrained model had a lower AIC value than the constrained model $(\triangle \mathrm{AIC}=19.76)$. 
Age-Related Changes in Negative Affect

Results are summarized in Table 2 and Figure 2 (see Supplementary Section 11 for Figure 2 with raw data points overlaid). For the general anxiety and sadness factors, the spline models produced a better fitting model than the linear model; thus, the spline models were selected for inference. General anxiety $(p=.021)$ and sadness $(p<.001)$ showed significant agerelated changes that could be described as "u" shaped, with slight decrease during childhood and sharper increases in adolescence. To explore these non-linear changes further, we conducted a "two-lines test" (Simonsohn, 2018; Nook et al., 2018) to determine whether the apparent u-shape was statistically reliable (see Supplementary Section 12 and accompanying text for details). We found evidence for a significant "u-shape" for both factors: when broken into two separate regressions, the curves showed a significant negative slope between ages 8 and 13 followed by a significant positive slope between the ages of 13 and 17. Calculations of the derivatives to identify significant age windows of change indicated that sadness increases significantly between the ages of 14.7 and 15.6 years. For the evaluative anxiety factor, the linear model produced a better fitting model than a spline model and was selected for inference. Evaluative anxiety was shown to increase linearly with increasing age $(\beta=.26, p<.001)$. Anger was not significantly associated with age $(\beta=.004, p=.913)$; thus, we interpret anger as remaining stable across our age range.

In our supplemental analyses, we found that 1) our factor structure is invariant across males and females and across age as indicated by two multi-group CFAs, (see Supplementary Section 13), 2) gender did not significantly moderate any of the observed affect - functioning relationships after correcting for multiple comparisons (see Supplementary Section 14), 3) evaluative anxiety and sadness show significant puberty-related changes in addition to age- 
related changes (see Supplementary Section 15), 4) emotional support and loneliness show significant, non-linear increases with age and perceived hostility shows significant, non-linear decreases with age (see Supplementary Section 16), and 5) general anxiety, evaluative anxiety, and anger show significant, specific relationships with DSM-derived domains from a dimensional self-report measure of psychopathology (see Supplementary Section 17).

\section{Discussion}

This study assessed distinct forms of negative affect and their relationship to social functioning, life satisfaction, and age in a large developmental sample. Four negative affect factors - general anxiety, anger, evaluative anxiety, and sadness- were extracted from an exploratory factor analysis, and fit indices from a confirmatory factor analysis suggested the model was plausible. General anxiety was associated with lower levels of emotional support and showed non-linear changes with age. Increased anger was associated with two types of maladaptive social functioning (perceived hostility and perceived rejection) and decreased life satisfaction and did not change significantly across the age range. Evaluative anxiety was associated with increased loneliness and increased linearly with increasing age. Sadness was significantly associated with all types of social functioning (emotional support, friendship, perceived hostility, loneliness, perceived rejection) and with decreased life satisfaction Sadness showed non-linear increases with age, with accelerated change between the ages of 14.7 and 15.6 years.

Taken together, our findings show an overall negative relationship between negative affect and daily functioning and an increase in negative affect from childhood into adolescence (see Supplementary Materials for an analysis of overall negative affect in our sample). These 
findings are largely consistent with previous work studying negative affect as one concept (e.g., Larson et al., 2002; Siedlecki et al. 2008). However, these relationships varied in strength across the four different forms of negative affect, as described below. By examining qualitatively different forms of negative affect within the broad positive-negative valence dimension, specific affect-functioning and affect-age relationships emerged.

General anxiety was significantly related to lower reported emotional support. This finding aligns with previous research in clinical populations that have demonstrated a link between anxiety and poorer social functioning in adolescence (de Lijster, 2018; La Greca, 1998; Biggs, 2012) and with work in adults showing negative correlations between trait anxiety and perceived social support (e.g., Hyde et al., 2011). Our findings also reveal that general anxiety declines slightly until around the transition to adolescence ( age 13) and then increases robustly during adolescence. Because the items in this factor describe a general anxiety without a specified cause (i.e., "I felt worried" rather than "I felt worried about..."), we speculate that the "u" shape we observe could be due to age-related differences in the source of the affective experience. For example, work done on normative samples of youth shows that separation anxiety and anxiety surrounding death and danger are predominant in children, whereas social anxiety and fear of failure and criticism are predominant in adolescence (Weems \& Costa, 2005). Individuals around age 13 may be at a developmental period where they are no longer experiencing anxieties of a child but for whom the social anxieties of an adolescent are just emerging. The increase in anxiety observed in adolescence also aligns with emotional development theories that suggest negative experiences such as anxiety and worry may increase as individuals transition into adolescence, as their world of concerns widens (e.g., anxiety associated with politics) (Larson \& Asmussen, 1991). 
Anger was significantly related to an increase in the perception of others' hostility and rejection. These two particular social domains are categorized as "social distress" by the NIH Toolbox (in contrast to other social domains of "perceived social support" or "companionship"), which is the extent to which an individual perceives his or her daily social interactions as negative or distressing. This link between anger and perceived rejection is consistent with previous research on "rejection sensitivity", or the tendency to anxiously or angrily expect or perceive rejection (Downey \& Feldman, 1996; Feldman \& Downey, 1994). Anger was also associated with reductions in life satisfaction, with aligns with previous research showing a link between these domains (e.g., Arrindell, 1991; Pilcher, 1998). Anger did not show significant age-related changes, indicating this affective experience remains relatively stable across the 817-year-old range examined in the current study.

Evaluative anxiety is a notable affective factor in that it consists of various affective states (i.e., worry, tension, and feelings of being hurt and upset) specifically in response to making mistakes, performing poorly, or being scolded. These items, originally from the BIS/BAS scale, are theorized to arise from a behavioral inhibition system in which the motivation is to avoid aversive outcomes (Carver \& White, 1994). In the clinical realm, this experience resembles social anxiety, which includes symptoms such as fear of social or performance situations in which one is exposed to scrutiny by others (American Psychiatric Association, 2013). Evaluative anxiety was significantly and positively related to the social domain of loneliness, which aligns with research showing close links between these two experiences (e.g., Johnson et al., 2001, Lim et al., 2016) Evaluative anxiety increased linearly with increasing age, which aligns with previous research showing increases across childhood and 
adolescence (Pagliaccio et al., 2016) and supports the possibility that with an emerging social understanding in adolescence comes an increase in evaluative concerns (Weems \& Costa, 2005).

While general anxiety, anger, and evaluative anxiety each showed specificity in their relationships to the outcome domains, sadness was globally related to all social functioning domains and to life satisfaction. While previous work does not comprehensively cover the sadness-functioning relationships investigated in this study, the relationships observed are overall consistent with previous work showing close links between sadness and functioning outcomes. For example, in a study examining adolescents' perceptions of loneliness, loneliness was positively associated with feelings of sadness (Buchholz \& Catton, 1999). In addition, the severity of depression symptoms, which is linked to experiences of sadness in adolescence (Chaplin, 2006), is strongly negatively correlated with life satisfaction (Headey et al., 1993). As with general anxiety, sadness declines slightly until around the transition to adolescence and then increases robustly during adolescence. Similar to general anxiety, this may be due to normative developmental differences in the cause and type of sadness experienced, especially in the context of changing social contexts (i.e., sadness from parents vs. sadness from peers or romantic relationships). However, our data cannot inform the cause for the observed age-related differences, and future research is needed to identify potential underlying mechanisms. We additionally find that sadness begins a period of particularly accelerated change at 14.7 years of age, which coincides with the median age of onset for any Diagnostic and Statistical Manual (DSM-IV) disorder (Kessler et al., 2005). These findings encourage translational research that investigates whether causal links exist between increasing sadness during mid adolescence and the onset of mental illness. 
Four limitations of the current study should be acknowledged. First, the data used in the current study are cross-sectional, which limits the inferences on the processes underlying the observed age-related changes in different forms of negative affect. A longitudinal design is needed to examine within-person changes in affect across adolescence, which could help identify emotion-related risk factors for the development of psychopathology. Second, the forms of negative affect observed were naturally limited by the range of items input to the EFA. While the items available in the current sample covered core affective categories such as anger, sadness, and fear, they did not include negative emotions such as guilt, embarrassment, or shame. Future studies should include a wider range of negative affect items to identify any additional existing forms of negative affect and their specific relationships to daily functioning. In addition, while we focused on affective experience, emotions are complex mental constructions that include additional levels that should be explored and characterized in adolescent samples (e.g., physiological responses, facial expressions, appraisals). Third, the data assessing associations between affect, social functioning, and life satisfaction are inherently correlational. Indeed, the emotional and social world of an adolescent is complex and the relationships between affective experiences, social functioning, and life satisfaction are likely bidirectional. Future work using experimental designs or longitudinal data with lead-lag analyses could permit more precise interpretations on the directionality of influence. Fourth, because participants age $18+$ were administered different items from children and adolescents, these older ages were not examined in the current study. Given the observed changes in negative affect toward the end of the adolescent age range studied and recent work suggesting mood disorders peak in early adulthood (Solmi et al., 2021), future research is needed to explore the age-related changes in forms of negative affect across this adolescent to adulthood transition. 
One additional promising area for future research is to investigate how qualitatively distinct forms of negative affect relate to brain connectivity in youth samples. Extensive research has shown that resting state and task-based functional connectivity is related to emotional processes in adolescent samples (Stevens, 2016 for review). However, little work has studied how qualitatively different negative affect states relate to connectivity profiles. Previous studies in adult samples suggest that distinct affective states have dissociable neural signatures (Petrican et al., 2015; Weathersby et al., 2019), but this should be investigated in developmental samples.

\section{Conclusion}

In this study we identified qualitatively different forms of negative affect in a developmental sample, and explored their relationship with social functioning, life satisfaction, and age. Results suggested that different forms of negative affect have distinct age-related changes and distinct associations with functioning outcomes, cautioning against studying negative affect as a singular concept and highlighting the importance of studying negative affect in this fine-grained way in youth samples. These findings motivate translational research to elucidate relationships between discrete negative affect forms and risk for psychopathology. 


\section{Figures}

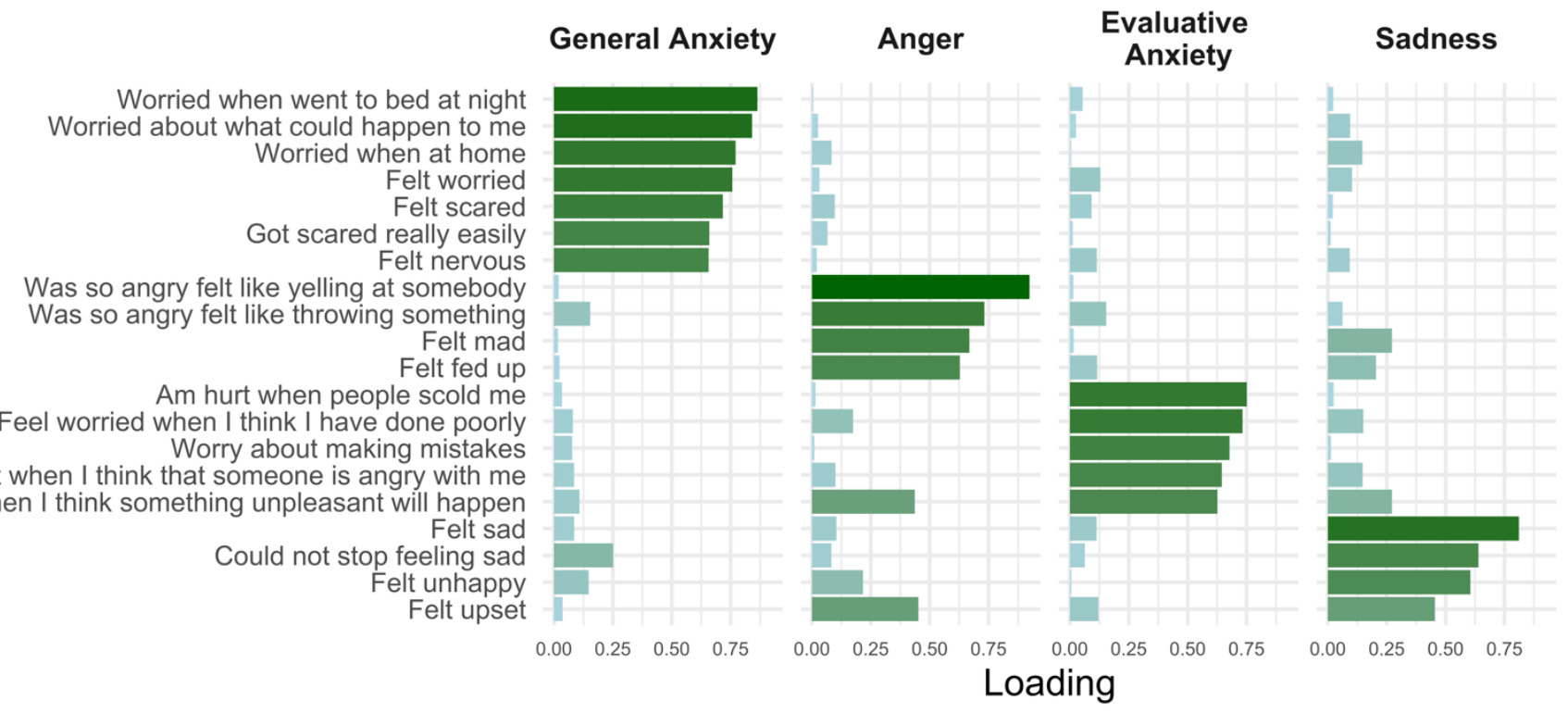

Figure 1. EFA loading plot. The absolute values of the loadings are plotted, sorted by loading strength. Higher loading strengths are depicted by larger values on the $\mathrm{x}$-axis and a darker green color. Items submitted to affect factor analysis are paraphrased on the left; the full items are available in the Supplementary Materials. 

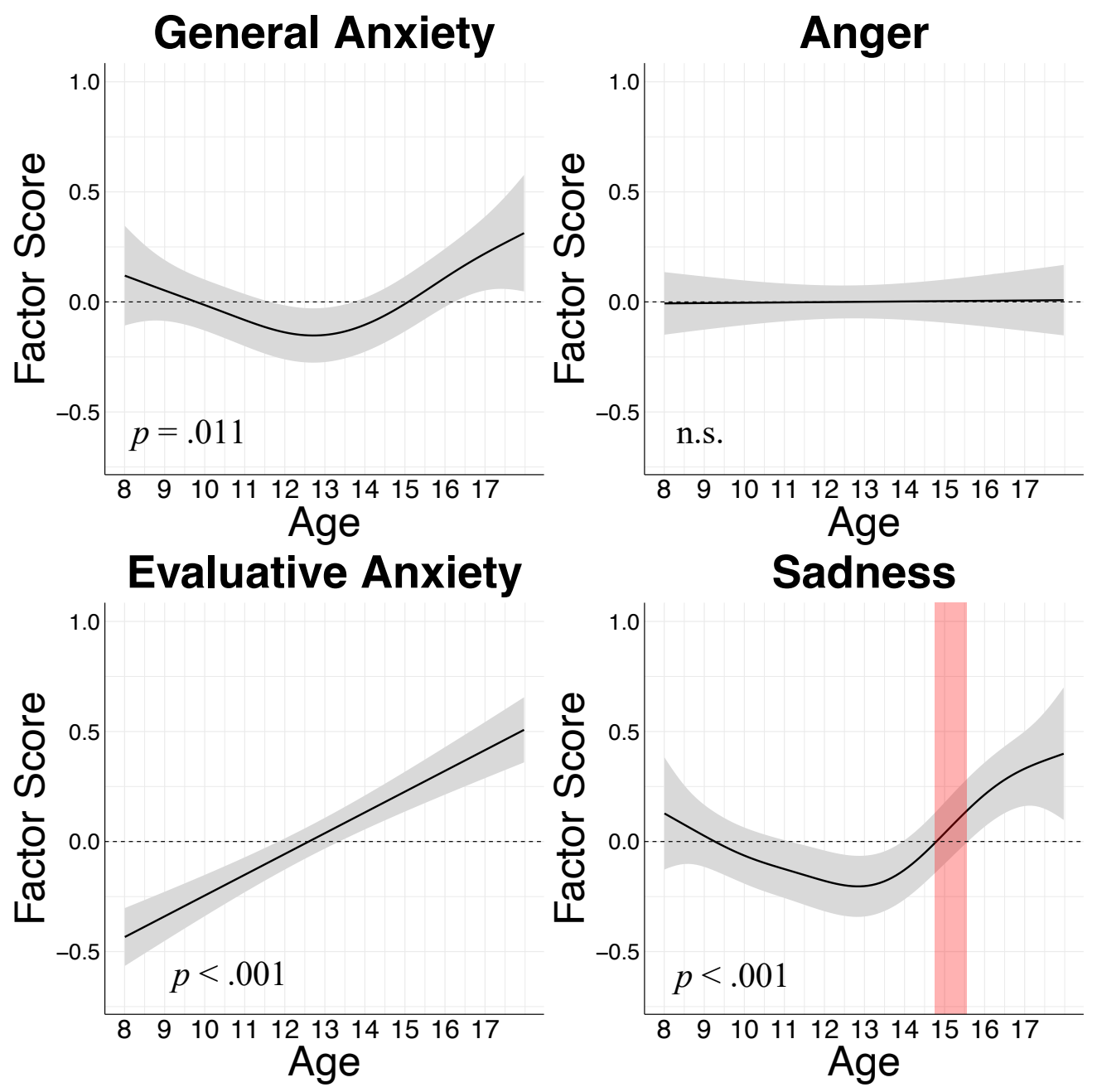

Figure 2. Age-Related Changes in Negative Affect Experience. Solid black line depicts linear or non-linear model fit. Grey shaded region depicts 95\% confidence interval. Red shaded region represents the window of accelerated change in the GAM. Y-axis units are factor scores. 


\section{Tables}

Table 1. Results of linear models for social functioning and life satisfaction dependent variables.

\begin{tabular}{|c|c|c|c|c|c|c|c|}
\hline Dependent variable & $\begin{array}{l}\text { Independent } \\
\text { variables }\end{array}$ & Coef & $95 \% \mathrm{CI}$ & $\begin{array}{c}\mathrm{t}- \\
\text { score }\end{array}$ & $\mathrm{df}$ & $\begin{array}{c}\mathrm{p}- \\
\text { value }\end{array}$ & $\begin{array}{c}\text { Corrected } \\
\text { p-value }\end{array}$ \\
\hline \multirow[t]{4}{*}{ Emotional Support } & General Anxiety & -0.16 & {$[-0.26,-0.06]$} & -3.15 & 763.06 & .002 & .024 \\
\hline & Anger & -0.06 & {$[-0.14,0.03]$} & -1.30 & 763.06 & .194 & .972 \\
\hline & Evaluative Anxiety & 0.07 & {$[-0.01,0.14]$} & 1.77 & 763.06 & .077 & .536 \\
\hline & Sadness & -0.16 & {$[-0.24,-0.07]$} & -3.50 & 763.06 & $<.001$ & .007 \\
\hline \multirow[t]{4}{*}{ Friendship } & General Anxiety & -0.04 & {$[-0.14,0.06]$} & -0.82 & 763.04 & .412 & 1.000 \\
\hline & Anger & -0.09 & {$[-0.18,0.00]$} & -2.00 & 763.04 & .046 & .447 \\
\hline & Evaluative Anxiety & -0.02 & {$[-0.10,0.05]$} & -0.55 & 763.04 & .583 & 1.000 \\
\hline & Sadness & -0.21 & {$[-0.30,-0.12]$} & -4.64 & 763.04 & $<.001$ & $<.001$ \\
\hline \multirow[t]{4}{*}{ Perceived Hostility } & General Anxiety & 0.08 & {$[-0.01,0.16]$} & 1.65 & 763.14 & .100 & .601 \\
\hline & Anger & 0.34 & {$[0.26,0.41]$} & 8.63 & 763.14 & $<.001$ & $<.001$ \\
\hline & Evaluative Anxiety & 0.08 & {$[0.01,0.14]$} & 2.32 & 763.14 & .021 & .247 \\
\hline & Sadness & 0.15 & {$[0.07,0.23]$} & 3.78 & 763.14 & $<.001$ & .003 \\
\hline \multirow[t]{4}{*}{ Loneliness } & General Anxiety & 0.08 & {$[0.00,0.17]$} & 2.00 & 764.00 & .046 & .447 \\
\hline & Anger & 0.07 & {$[0.00,0.14]$} & 2.01 & 764.00 & .045 & .447 \\
\hline & Evaluative Anxiety & 0.14 & {$[0.08,0.20]$} & 4.36 & 764.00 & $<.001$ & $<.001$ \\
\hline & Sadness & 0.47 & {$[0.40,0.55]$} & 12.75 & 764.00 & $<.001$ & $<.001$ \\
\hline \multirow[t]{4}{*}{ Perceived Rejection } & General Anxiety & 0.12 & {$[0.04,0.21]$} & 2.75 & 761.80 & .006 & .080 \\
\hline & Anger & 0.23 & {$[0.15,0.30]$} & 5.88 & 761.80 & $<.001$ & $<.001$ \\
\hline & Evaluative Anxiety & 0.08 & {$[0.01,0.14]$} & 2.27 & 761.80 & .023 & .258 \\
\hline & Sadness & 0.28 & {$[0.20,0.36]$} & 6.99 & 761.80 & $<.001$ & $<.001$ \\
\hline \multirow[t]{4}{*}{ Life Satisfaction } & General Anxiety & 0.03 & {$[-0.09,0.14]$} & 0.46 & 535.60 & .648 & 1.000 \\
\hline & Anger & -0.22 & {$[-0.32,-0.12]$} & -4.46 & 535.60 & $<.001$ & $<.001$ \\
\hline & Evaluative Anxiety & 0.02 & {$[-0.06,0.10]$} & 0.44 & 535.60 & .657 & 1.000 \\
\hline & Sadness & -0.28 & {$[-0.38,-0.18]$} & -5.39 & 535.60 & $<.001$ & $<.001$ \\
\hline \multicolumn{8}{|c|}{$\begin{array}{l}\text { Note: Coef: standardized regression coefficient; CI: Confidence Interval. P-values were corrected using } \\
\text { the Holm method ( } 6 \text { total regressions each with } 4 \text { affect factors }=24 \text { total tests) and were compared to an } \\
\text { alpha level of } .05 \text { to determine significance. Bold text indicates significant models. Spline age was } \\
\text { controlled for in all models. }\end{array}$} \\
\hline
\end{tabular}


Table 2. Results of linear and thin plate smoothing spline models for each dependent variable assessing age related change.

\begin{tabular}{lcccc}
\hline Dependent variable & Model & AIC & p-value & Corrected p-value \\
\hline General Anxiety & Linear & 2256.15 & & \\
& Spline & $\mathbf{2 2 4 6 . 2 6}$ & $\mathbf{. 0 1 2}$ & $\mathbf{. 0 2 1}$ \\
Anger & Linear & 2274.06 & .913 & .913 \\
& Spline & 2274.06 & & \\
Sadness & Linear & 2208.65 & & \\
& Spline & $\mathbf{2 1 9 3 . 3 8}$ & $\mathbf{< . 0 0 1}$ & $\mathbf{< . 0 0 1}$ \\
\multirow{2}{*}{ Evaluative Anxiety } & Linear & $\mathbf{2 1 5 4 . 0 2}$ & $\mathbf{< . 0 0 1}$ & $\mathbf{< . 0 0 1}$ \\
& Spline & 2154.02 & & \\
\hline
\end{tabular}

Note: Bold text indicates best fitting model for each dependent variable, as determined by AIC. AIC $=$ Akaike Information Criterion. For spline models, p-value refers to approximate significance of smooth age terms. For linear models, $p$-value refers to significance of age coefficient. P-values were corrected using the Holm method and were compared to an alpha level of .05 to determine significance. 


\section{References}

Achenbach, T. M. (1997). Manual for the young adult self-report and young adult behavior checklist. University of Vermont, Department of Psychiatry.

American Psychiatric Association. (2013). Diagnostic and statistical manual of mental disorders (DSM-5®). American Psychiatric Pub.

Arrindell, W. A., Meeuwesen, L., \& Huyse, F. J. (1991). The Satisfaction With Life Scale (SWLS): Psychometric properties in a non-psychiatric medical outpatients sample. Personality and individual differences, 12(2), 117-123.

Biggs, B. K., Vernberg, E. M., \& Wu, Y. P. (2012). Social anxiety and adolescents' friendships: The role of social withdrawal. The Journal of Early Adolescence, 32(6), 802-823.

Buchholz, E. S., \& Catton, R. (1999). Adolescents' perceptions of aloneness and loneliness. Adolescence, 34(133), 203-204.

Carver, C. S., \& White, T. L. (1994). Behavioral inhibition, behavioral activation, and affective responses to impending reward and punishment: The BIS/BAS scales. Journal of Personality and Social Psychology, 67, 319-333

Chaplin, T. M. (2006). Anger, happiness, and sadness: Associations with depressive symptoms in late adolescence. Journal of Youth and Adolescence, 35(6), 977-986.

Chen, F. F. (2007). Sensitivity of goodness of fit indexes to lack of measurement invariance. Structural equation modeling: a multidisciplinary journal, 14(3), 464-504.

Cheung, G. W., \& Rensvold, R. B. (2002). Evaluating goodness-of-fit indexes for testing measurement invariance. Structural equation modeling, 9(2), 233-255.

Chida, Y., \& Steptoe, A. (2008). Positive psychological well-being and mortality: a quantitative review of prospective observational studies. Psychosomatic medicine, 70(7), 741-756. 
Csardi G., Nepusz T. (2006). The igraph software package for complex network research, InterJournal, Complex Systems 1695. http://igraph.org

de Lijster, J. M., Dieleman, G. C., Utens, E. M., Dierckx, B., Wierenga, M., Verhulst, F. C., \& Legerstee, J. S. (2018). Social and academic functioning in adolescents with anxiety disorders: A systematic review. Journal of affective disorders, 230, 108-117.

Diener, E. (1984). Subjective Well-Being. Psychological Bulletin, 95(3), 542-575.

Downey, G., \& Feldman, S. I. (1996). Implications of rejection sensitivity for intimate relationships. Journal of Personality and Social Psychology, 70, 1327 - 1343.

Feldman, S., \& Downey, G. (1994). Rejection sensitivity as a mediator of the impact of childhood exposure to family violence on adult attachment behavior. Development and Psychopathology, 6, $231-247$.

Forbes, E. E., Williamson, D. E., Ryan, N. D., \& Dahl, R. E. (2004). Positive and negative affect in depression: Influence of sex and puberty. Annals of the New York Academy of Sciences, 1021(1), 341-347.

Gatignon, H. (2010). Confirmatory factor analysis. In Statistical analysis of management data (pp. 59-122). Springer, New York, NY.

Gershon, R. C., Wagster, M. V., Hendrie, H. C., Fox, N. A., Cook, K. F., \& Nowinski, C. J. (2013). NIH toolbox for assessment of neurological and behavioral function. Neurology, 80(11 Supplement 3), S2-S6.

Grisanzio, K. (2021, April 27). Shifting qualities of negative affective experience through adolescence: Associations with functional outcomes. Retrieved from osf.io/dsxnu Headey, B., Kelley, J., \& Wearing, A. (1993). Dimensions of mental health: Life satisfaction, positive affect, anxiety, and depression. Social Indicators Research, 29, 63-82. 
Holm, S. (1979). A simple sequentially rejective multiple test procedure. Scandinavian Journal of Statistics, 6, 65-70.

Hu, L., \& Bentler, P. M. (1999). Cutoff criteria for fit indexes in covariance structure analysis: Conventional criteria versus new alternatives. Structural Equation Modeling, 6, 1-55.

Hyde, L. W., Gorka, A., Manuck, S. B., \& Hariri, A. R. (2011). Perceived social support moderates the link between threat-related amygdala reactivity and trait anxiety. Neuropsychologia, 49(4), 651-656.

Johnson, H. D., Lavoie, J. C., \& Mahoney, M. (2001). Interparental conflict and family cohesion: Predictors of loneliness, social anxiety, and social avoidance in late adolescence. Journal of adolescent research, 16(3), 304-318.

Kessler, R. C., Berglund, P., Demler, O., Jin, R., Merikangas, K. R., \& Walters, E. E. (2005). Lifetime prevalence and age-of-onset distributions of DSM-IV disorders in the National Comorbidity Survey Replication. Archives of general psychiatry, 62(6), 593-602.

La Greca, A. M., \& Lopez, N. (1998). Social anxiety among adolescents: Linkages with peer relations and friendships. Journal of abnormal child psychology, 26(2), 83-94.

Larson, R. W., Moneta, G., Richards, M. H., \& Wilson, S. (2002). Continuity, stability, and change in daily emotional experience across adolescence. Child Development, 73(4), 1151-1165.

Larson, R. W., \& Richards, M. H. (2000). Changes in daily emotions associated with entry into adolescence for urban African Americans. In biannual meeting of the International Society for the Study of behavioral development.

Larson, R., \& Asmussen, L. (1991). Anger, worry, and hurt in early adolescence: An enlarging world of negative emotions. Adolescent stress: Causes and consequences, 21-41. 
Larson, R. W., Raffaelli, M., Richards, M. H., Ham, M., \& Jewell, L. (1990). Ecology of depression in late childhood and early adolescence: A profile of daily states and activities. Journal of Abnormal Psychology, 99(1), 92.

Lee, F. S., Heimer, H., Giedd, J. N., Lein, E. S., Šestan, N., Weinberger, D. R., \& Casey, B. J. (2014). Adolescent mental health-opportunity and obligation. Science, 346(6209), 547549. doi: $10.1126 /$ science. 1260497

Lim, M. H., Rodebaugh, T. L., Zyphur, M. J., \& Gleeson, J. F. (2016). Loneliness over time: The crucial role of social anxiety. Journal of abnormal psychology, 125(5), 620.

Moneta, G. B., Schneider, B., \& Csikszentmihalyi, M. (2001). A longitudinal study of the selfconcept and experiential components of self-worth and affect across adolescence. Applied Developmental Science, 5(3), 125-142.

Nook, E. C., Sasse, S. F., Lambert, H. K., McLaughlin, K. A., \& Somerville, L. H. (2018). The nonlinear development of emotion differentiation: Granular emotional experience is low in adolescence. Psychological science, 29(8), 1346-1357.

Pagliaccio, D., Luking, K. R., Anokhin, A. P., Gotlib, I. H., Hayden, E. P., Olino, T. M., Peng, C., Hajcak, G. \& Barch, D. M. (2016). Revising the BIS/BAS Scale to study development: Measurement invariance and normative effects of age and sex from childhood through adulthood. Psychological assessment, 28(4), 429.

Paolini, L., Yanez, A. P., \& Kelly, W. E. (2006). An Examination of Worry and Life Satisfaction Among College Students. Individual Differences Research, 4(5).

Pavot, W., \& Diener, E. (2008). The satisfaction with life scale and the emerging construct of life satisfaction. The journal of positive psychology, 3(2), 137-152. 
Patton, G. C., Coffey, C., Romaniuk, H., Mackinnon, A., Carlin, J. B., Degenhardt, L., Olsson, C. A., \& Moran, P. (2014). The prognosis of common mental disorders in adolescents: a 14-year prospective cohort study. The Lancet, 383(9926), 1404-1411.

Petrican, R., Saverino, C., Rosenbaum, R. S., \& Grady, C. (2015). Inter-individual differences in the experience of negative emotion predict variations in functional brain architecture. Neuroimage, 123, 80-88.

Petersen, A. C., Crockett, L., Richards, M., \& Boxer, A. (1988). A self-report measure of pubertal status: Reliability, validity, and initial norms. Journal of Youth and Adolescence, $17(2), 117-133$.

Pilcher, J. J. (1998). Affective and daily event predictors of life satisfaction in college students. Social indicators research, 43(3), 291-306.

R Core Team (2020). R: A language and environment for statistical computing. R Foundation for Statistical Computing, Vienna, Austria. URL https://www.R-project.org/.

Revelle, W. (2015). An introduction to psychometric theory with applications in R. Freely available online. http://www.personality-project.org/r/book/

Revelle, W. (2018). psych: Procedures for Personality and Psychological Research, Northwestern University, Evanston, Illinois, USA, https://CRAN.Rproject.org/package=psych Version $=1.8 .12$.

Rodman, A. M., Powers, K. E., \& Somerville, L. H. (2017). Development of self-protective biases in response to social evaluative feedback. Proceedings of the National Academy of Sciences, 114(50), 13158-13163.

Rosseel Y (2012). “lavaan: An R Package for Structural Equation Modeling.” Journal of Statistical Software, 48(2), 1-36. http://www.jstatsoft.org/v48/i02/. 
Rudolph, K. D., \& Hammen, C. (1999). Age and gender as determinants of stress exposure, generation, and reactions in youngsters: A transactional perspective. Child development, 70(3), 660-677.

Rudolph, K. D., Hammen, C., Burge, D., Lindberg, N., Herzberg, D., \& Daley, S. E. (2000). Toward an interpersonal life-stress model of depression: The developmental context of stress generation. Development and psychopathology, 12(2), 215-234.

Ruppert, D., Wand, M. P., and Carroll, R. J. (2003). Semiparametric regression. Cambridge University Press.

Russell, J. A. (1980). A circumplex model of affect. Journal of personality and social psychology, 39(6), 1161.

Silk, J. S., Steinberg, L., \& Morris, A. S. (2003). Adolescents' emotion regulation in daily life: Links to depressive symptoms and problem behavior. Child development, 74(6), 18691880.

Siedlecki, K. L., Tucker-Drob, E. M., Oishi, S., \& Salthouse, T. A. (2008). Life satisfaction across adulthood: Different determinants at different ages? The Journal of Positive Psychology, 3(3), 153-164.

Simonsohn, U. (2018). Two lines: A valid alternative to the invalid testing of U-shaped relationships with quadratic regressions. Advances in Methods and Practices in Psychological Science, 1(4), 538-555.

Simpson, G. L. (2020). gratia: Graceful 'ggplot'-Based Graphics and Other Functions for GAMs Fitted Using 'mgcv'. R package version 0.4.1. https:/CRAN.Rproject.org/package=gratia Solmi, M., Radua, J., Olivola, M., Croce, E., Soardo, L., de Pablo, G. S., Shin, J. I., Kirkbride, J. B., Jones, P., Kim, J. H., Kim, J. Y., Carvalho, A. F., Seeman, M. V., Correll, C. U., \& 
Fusar-Poli, P. (2021). Age at onset of mental disorders worldwide: large-scale metaanalysis of 192 epidemiological studies. Molecular psychiatry, 1-15.

Somerville, L. H. (2013). The teenage brain: Sensitivity to social evaluation. Current Directions in Psychological Science, 22(2), 129-135.

Somerville, L. H. (2016). Emotional development in adolescence. In Handbook of Emotion, 4th Edition (Barrett, Lewis, \& Haviland-Jones, Eds.).

Somerville, L. H., Bookheimer, S. Y., Buckner, R. L., Burgess, G. C., Curtiss, S. W., Dapretto, M., Elam, J. S., Gaffrey, M. S., Harms, M. P., Hodge, C. \& Kandala, S. (2018). The Lifespan Human Connectome Project in Development: A large-scale study of brain connectivity development in 5-21 year olds. Neuroimage, 183, 456-468.

Stevens, M. C. (2016). The contributions of resting state and task-based functional connectivity studies to our understanding of adolescent brain network maturation. Neuroscience \& Biobehavioral Reviews, 70, 13-32.

Thompson, B. L., Waltz, J., Croyle, K., \& Pepper, A. C. (2007). Trait meta-mood and affect as predictors of somatic symptoms and life satisfaction. Personality and individual Differences, 43(7), 1786-1795.

Watson, D., Clark, L. A., \& Stasik, S. M. (2011). Emotions and the emotional disorders: A quantitative hierarchical perspective. International Journal of Clinical and Health Psychology, 11(3), 429-442.

Weathersby, F. L., King, J. B., Fox, J. C., Loret, A., \& Anderson, J. S. (2019). Functional connectivity of emotional well-being: Overconnectivity between default and attentional networks is associated with attitudes of anger and aggression. Psychiatry Research: Neuroimaging, 291, 52-62. 
Weems, C. F., \& Costa, N. M. (2005). Developmental differences in the expression of childhood anxiety symptoms and fears. Journal of the American Academy of Child \& Adolescent Psychiatry, 44(7), 656-663.

Wood, S. N. (2003). Thin plate regression splines. Journal of the Royal Statistical Society B, $65(1), 95-114$.

Wood, S.N. (2017) Generalized Additive Models: An Introduction with R (2nd edition). Chapman and Hall/CRC. 


\title{
Supplementary Materials
}

\author{
To accompany
}

\section{Shifting qualities of negative affective experience through adolescence: Age-related change and associations with functional outcomes}

Contents

1. Selection of Final Sample (Supplementary Figure 1)

2. Age Distribution of Sample (Supplementary Figure 2)

3. Inclusion and exclusion criteria (Supplementary Table 1)

4. Included data by research site (Supplementary Table 2)

5. Negative affect items and data-driven factor assignment (Supplementary Table 3)

6. Exploratory factor analysis stability (Supplementary Figure 3, 4)

7. Exploratory factor analysis solution comparisons (Supplementary Figure 5; Supplementary Table 4)

8. Negative affect as a summary score (Supplementary Table 5, Supplementary Figure 6)

9. Scree plot for primary 4-factor solution (Supplementary Figure 7)

10. Confirmatory Factor Analysis parameter estimates (Supplementary Table 6)

11. Age-related changes in negative affect factors (raw data points overlaid)

12. Two-lines test to investigate nonlinear "u-shape" curves (Supplementary Figure 9)

13. Multi-group CFA to identify whether the factor structure is invariant across gender and age bins (Supplementary Table 7)

14. Testing gender as a moderator between negative affect factors and functioning outcomes (Supplementary Table 8; Supplementary Figure 10)

15. Negative affect changes as a function of pubertal development (Supplementary Table 9; Supplementary Figure 11)

16. Age-related changes in the social functioning and life satisfaction outcome domains (Supplementary Figure 12)

17. Exploration of the relationship between negative affect factors and clinically relevant measures (Supplementary Table 10) 


\section{Selection of Final Sample}

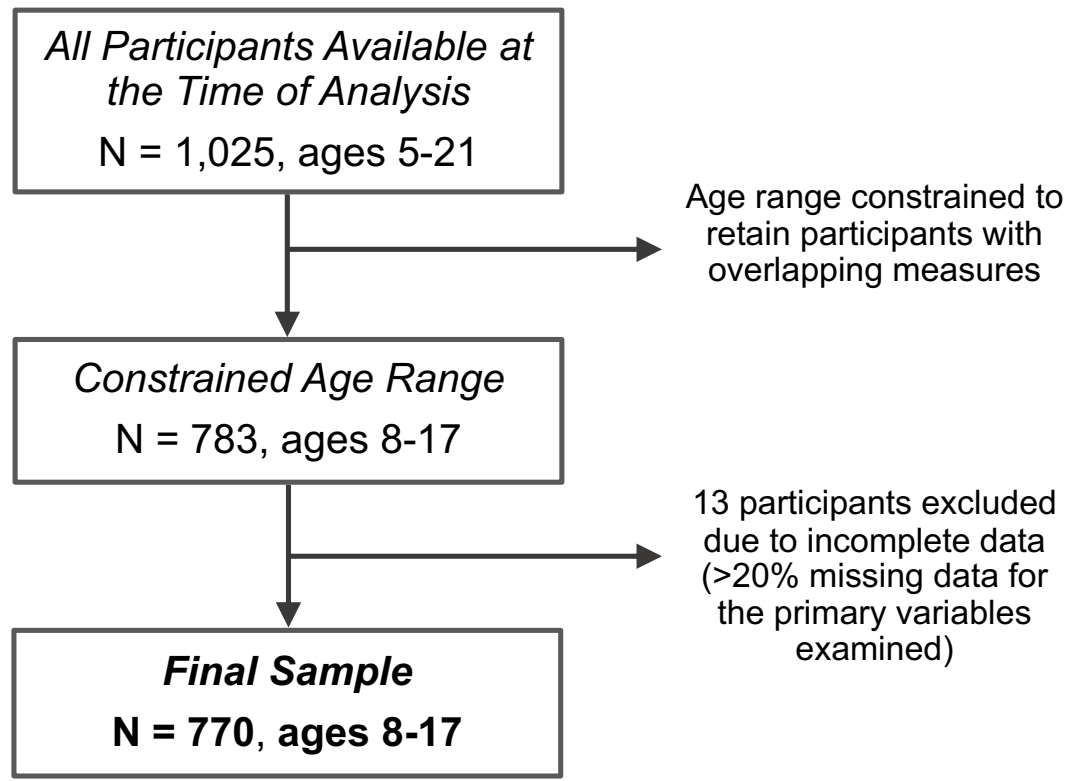

Supplementary Figure 1. Flow chart showing the selection of the final sample analyzed in the study. 


\section{Age Distribution of Sample}

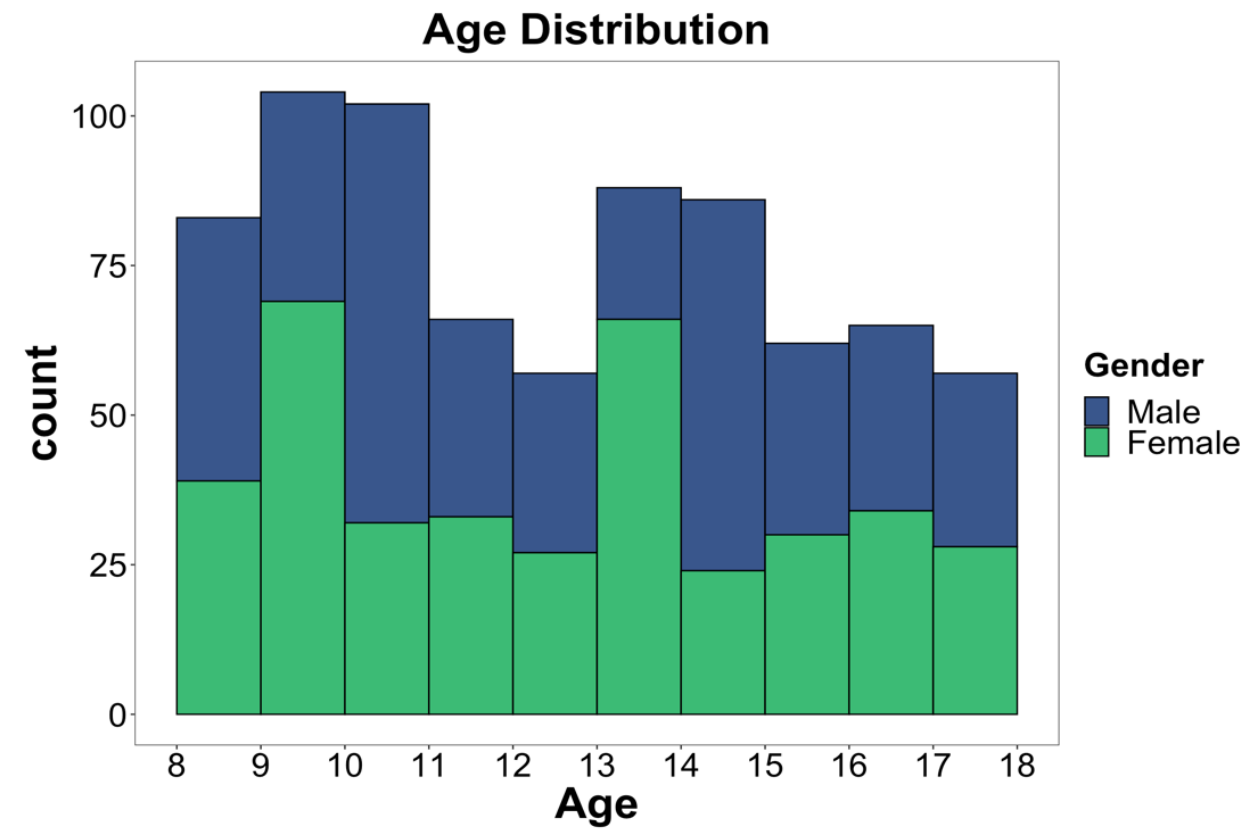

Supplementary Figure 2. Blue bars depict number of male participants, green bars depict number of female participants. 


\section{Inclusion and exclusion criteria}

Supplementary Table 1. Overview of inclusion and exclusion criteria used in the study (Somerville et al., 2018).

\begin{tabular}{l|l}
\hline Inclusion Criteria & Exclusion Criteria \\
\hline Age 5-21 years & Premature birth \\
\hline Speaks English well & Serious medical conditions (e.g., stroke, cerebral palsy) \\
\hline Safe to enter MRI & $\begin{array}{l}\text { Serious endocrine condition (e.g., precocious puberty, untreated growth } \\
\text { hormone deficiency) }\end{array}$ \\
\hline & Long term use of immunosuppressants or steroids \\
\cline { 2 - 2 } & Any history of serious head injury \\
\cline { 2 - 2 } & $\begin{array}{l}\text { Hospitalization }>2 \text { days for certain physical or psychiatric conditions or } \\
\text { substance use }\end{array}$ \\
\cline { 2 - 2 } & Treatment $>12$ months for psychiatric conditions \\
\cline { 2 - 2 } & Receiving certain special services at school \\
\cline { 2 - 2 } & Claustrophobia \\
\cline { 2 - 2 } & Pregnancy \\
\hline
\end{tabular}




\section{Included data by research site}

Supplementary Table 2. Participant age and gender by research site.

\begin{tabular}{lllll} 
& Harvard & UCLA & UMinn & WUSTL \\
\hline Total N & 262 & 162 & 188 & 158 \\
\hline Age (mean years (SD)) & $12.51(2.97)$ & $12.70(2.59)$ & $12.78(2.94)$ & $12.44(2.77)$ \\
\hline Gender (number $(\%)$ female) & $132(50.4 \%)$ & $77(47.5 \%)$ & $95(50.5 \%)$ & $78(49.4 \%)$ \\
\hline
\end{tabular}

Note: Data was collected at four research sites across the United States: Harvard University, University of California Los Angeles (UCLA) University of Minnesota (UMinn), and Washington University in St. Louis (WUSTL). 


\section{Negative affect items and data-driven factor assignment}

Supplementary Table 3. Final selection of negative affect items, the original measure they were selected from, and their factor assignment.

\begin{tabular}{|c|c|c|c|}
\hline Full Item & Abbreviation & Measure & $\begin{array}{l}\text { Factor } \\
\text { Assignment }\end{array}$ \\
\hline I felt mad & PedRepAng13 & NIH Toolbox Anger Subscale & Anger \\
\hline $\begin{array}{l}\text { I was so angry I felt like yelling at } \\
\text { somebody }\end{array}$ & PedRepAng14 & NIH Toolbox Anger Subscale & Anger \\
\hline I felt fed up & PedRepAng16 & NIH Toolbox Anger Subscale & Anger \\
\hline $\begin{array}{l}\text { I was so angry I felt like throwing } \\
\text { something }\end{array}$ & PedRepAng17 & NIH Toolbox Anger Subscale & Anger \\
\hline I felt upset & PedRepAng18 & NIH Toolbox Anger Subscale & Sadness \\
\hline I felt scared & PedRepAnx42 & NIH Toolbox Fear Subscale & General anxiety \\
\hline $\begin{array}{l}\text { I worried about what could happen } \\
\text { to me }\end{array}$ & PedRepAnx43 & NIH Toolbox Fear Subscale & General anxiety \\
\hline I felt worried & PedRepAnx44 & NIH Toolbox Fear Subscale & General anxiety \\
\hline I worried when I went to bed at night & PedRepAnx46 & NIH Toolbox Fear Subscale & General anxiety \\
\hline I felt nervous & PedRepAnx48 & NIH Toolbox Fear Subscale & General anxiety \\
\hline I worried when I was at home & PedRepAnx50 & NIH Toolbox Fear Subscale & General anxiety \\
\hline I got scared really easily & PedRepAnx51 & NIH Toolbox Fear Subscale & General anxiety \\
\hline I felt unhappy & PedRepDep36 & $\begin{array}{l}\text { NIH Toolbox Sadness } \\
\text { Subscale }\end{array}$ & Sadness \\
\hline I felt sad & PedRepDep38 & $\begin{array}{l}\text { NIH Toolbox Sadness } \\
\text { Subscale }\end{array}$ & Sadness \\
\hline I could not stop feeling sad & PedRepDep41 & $\begin{array}{l}\text { NIH Toolbox Sadness } \\
\text { Subscale }\end{array}$ & Sadness \\
\hline $\begin{array}{l}\text { I usually get very tense when I think } \\
\text { something unpleasant is going to } \\
\text { happen }\end{array}$ & bisbas 1 & BIS/BAS Scale & $\begin{array}{l}\text { Evaluative } \\
\text { anxiety }\end{array}$ \\
\hline I worry about making mistakes & bisbas2 & BIS/BAS Scale & $\begin{array}{l}\text { Evaluative } \\
\text { anxiety }\end{array}$ \\
\hline $\begin{array}{l}\text { I am hurt when people scold me or } \\
\text { tell me that I do something wrong }\end{array}$ & bisbas3 & BIS/BAS Scale & $\begin{array}{l}\text { Evaluative } \\
\text { anxiety }\end{array}$ \\
\hline $\begin{array}{l}\text { I feel pretty upset when I think that } \\
\text { someone is angry with me }\end{array}$ & bisbas 4 & BIS/BAS Scale & $\begin{array}{l}\text { Evaluative } \\
\text { anxiety }\end{array}$ \\
\hline $\begin{array}{l}\text { I do not become fearful or nervous, } \\
\text { even when something bad happens to } \\
\text { me }\end{array}$ & bisbas5 & BIS/BAS Scale & N/A \\
\hline $\begin{array}{l}\text { I feel worried when I think I have } \\
\text { done poorly at something }\end{array}$ & bisbas6 & BIS/BAS Scale & $\begin{array}{l}\text { Evaluative } \\
\text { anxiety }\end{array}$ \\
\hline $\begin{array}{l}\text { I am very fearful compared to my } \\
\text { friends }\end{array}$ & bisbas 7 & BIS/BAS Scale & $\mathrm{N} / \mathrm{A}$ \\
\hline
\end{tabular}




\section{Exploratory factor analysis stability}

To examine the stability of the EFA solution, we ran the same EFA methods described in the manuscript across 1,000 bootstrapped samples. For each item in each iteration, we retained the highest loading and visualized the consistency of these maximum loadings across the bootstrapped samples (Supplementary Figure 3).

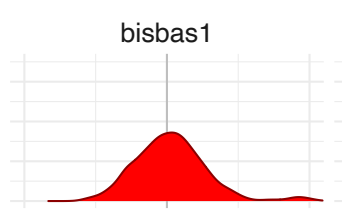

bisbas6

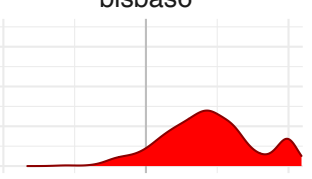

PedRepAng17

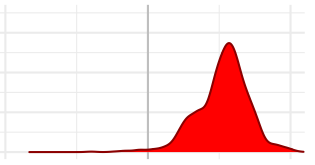

PedRepAnx46

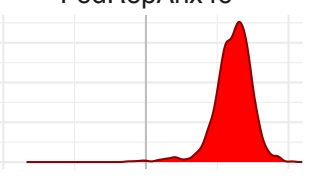

PedRepDep38

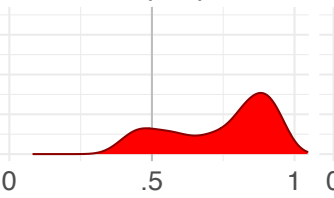

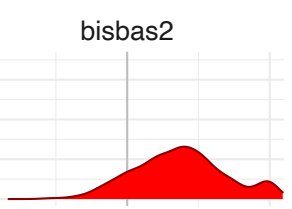

bisbas 7

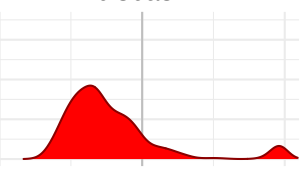

PedRepAng18

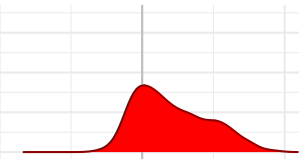

PedRepAnx48

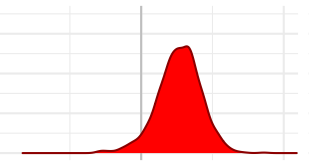

PedRepDep41

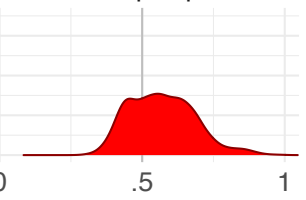

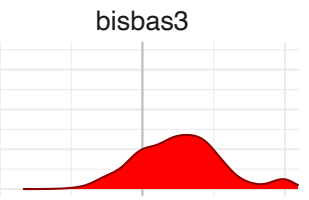

PedRepAng13

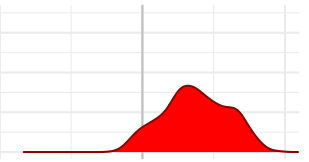

PedRepAnx42

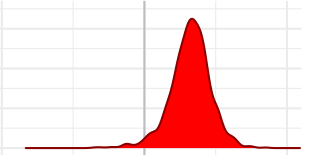

PedRepAnx50

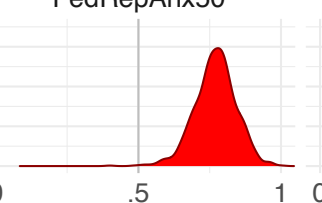

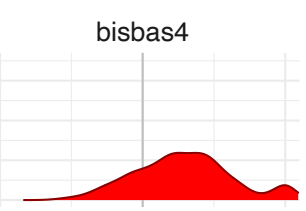

PedRepAng14

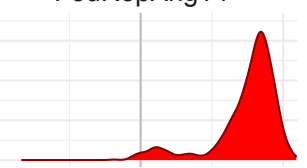

PedRepAnx43

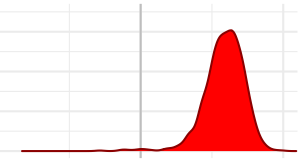

PedRepAnx51

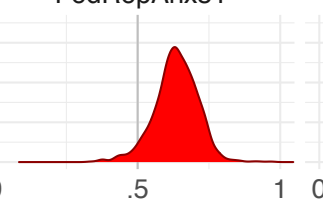

.5

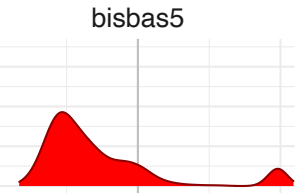

PedRepAng16

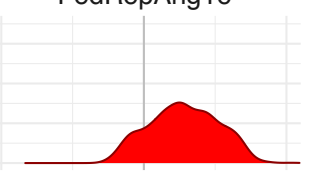

PedRepAnx44

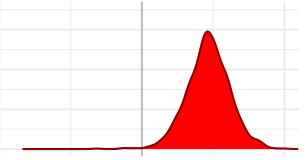

PedRepDep36

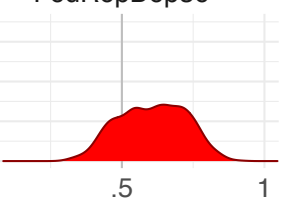

Maximum Loading

Supplementary Figure 3. Consistency of maximum loadings. Each plot depicts a histogram of the maximum loadings each item achieved on any factor across the 1,000 bootstrapped samples. For full item names see Supplementary Table 3.

As shown in Supplementary Figure 3, items "bisbas5" and "bisbas7" consistently had low loadings of less than .5 , thus, to improve the stability of our factor solution, we removed these items for all primary analyses. 
Second, we visualized how often items shared the same factor for their primary loading across the bootstrap iterations. This method is not sensitive to differences in arbitrary dimension rotations (e.g., that factor 1 in iteration 1 becomes factor 4 in iteration 2). We built up an adjacency matrix of these counts (see code at https://osf.io/dsxnu/) and visualize it as a graph using the igraph package (Csardi \& Nepusz, 2006; Supplementary Figure 4).

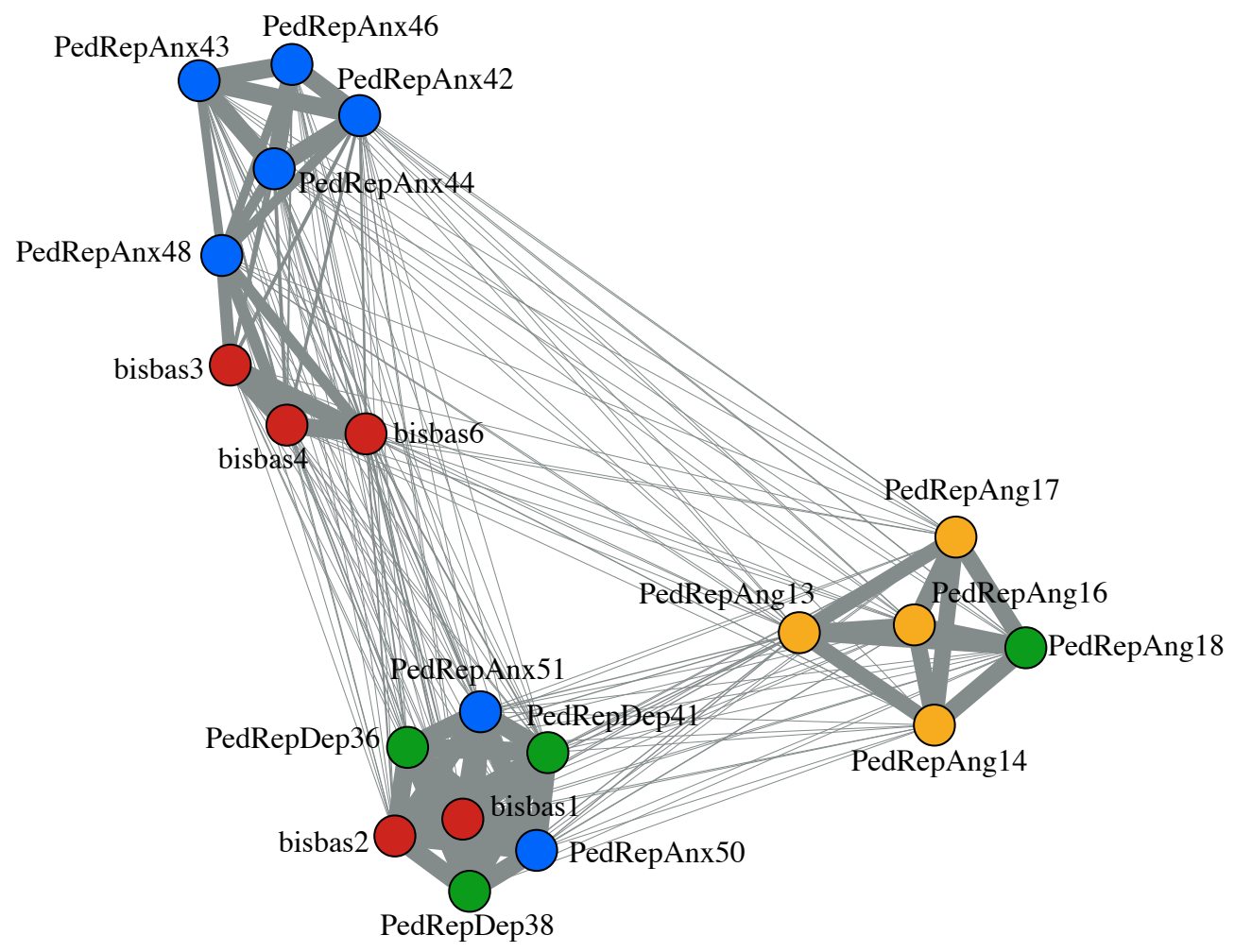

Supplementary Figure 4. Stability of factor solution. Nodes represent the twenty items used in the EFA. Edge weights between nodes show how frequently two items load onto the same factor across 1,000 bootstrapped samples. Colors correspond to the final 4-factor solution reported in the manuscript (blue $=$ general anxiety, yellow $=$ anger, green $=$ sadness, red $=$ evaluative anxiety. For full item names see Supplementary Table 3.

Supplementary Figure 4 shows that with the exception of the cluster of items at the bottom of the figure, the items in the final factor solution generally cluster together. For example, the four items that ultimately form our anger factor are shown to cluster together in 
Supplementary Figure 5 (yellow dots), indicating that over 1,000 bootstrapped samples, they tended to load onto the same factor. The item PedRepAng18 ("I felt upset", green dot) loaded onto the sadness factor more strongly in the final solution, but here is shown to load with other anger items across bootstrapped samples. The items that ultimately loaded onto the general anxiety factor also tended to load together across the bootstrapped samples (i.e., the cluster of blue items at the top of the figure), with the exception of "PedRepAnx51" and "PedRepAnx50" (shown in the mixed cluster at the bottom of the figure). Similarly, the items that ultimately loaded onto the evaluative anxiety factor tended to load together across bootstrapped samples (i.e., the cluster of red items in the top left of the figure), with the exception of "bisbas 1" and "bisbas2" (shown in the mixed cluster at the bottom of the figure). Overall, Supplementary Figure 5 shows a possible alternate 3 -factor solution (i.e., the blue and red items in the top left as one factor, the bottom cluster as one factor, and the right cluster as one factor). To quantitatively compare this possible factor solution with the EFA-derived solution reported in the manuscript, we calculate and report CFA fit statistics for both models (see the following section, Supplementary Materials, Section 7), and find that the solution generated by the EFA (reported in the manuscript) is a better fitting model. 


\section{Exploratory factor analysis solution comparisons}

To ensure the 4-factor EFA solution identified in the manuscript is the best fitting solution to represent the latent structure of the negative affect variables, we compared our solution with three additional proposed factor structures: 1) an EFA-derived solution using all twenty-two negative affect items (including "bisbas5" and "bisbas7", which were removed due to consistently low loadings), 2) a 3-factor structure matching the three visual groupings of items in Supplementary Figure 5, and 3) due to potential theoretical similarity between the general anxiety and evaluative anxiety factors, a 3-factor solution combining items from these two factors (resulting factors being anxiety, anger, and sadness). The EFA conducted on all twenty-two negative affect items was run using the same methods as reported in the manuscript and yielded the same 4 factors as the solution reported on in the manuscript, with the exception of the item "I felt upset" loading more strongly onto the anger factor than the sadness factor in this solution. Items "bisbas5" ("Do not become fearful or nervous") and "bisbas7" ("Fearful compared to friends") loading weakly onto the general anxiety factor (Supplementary Figure 5).

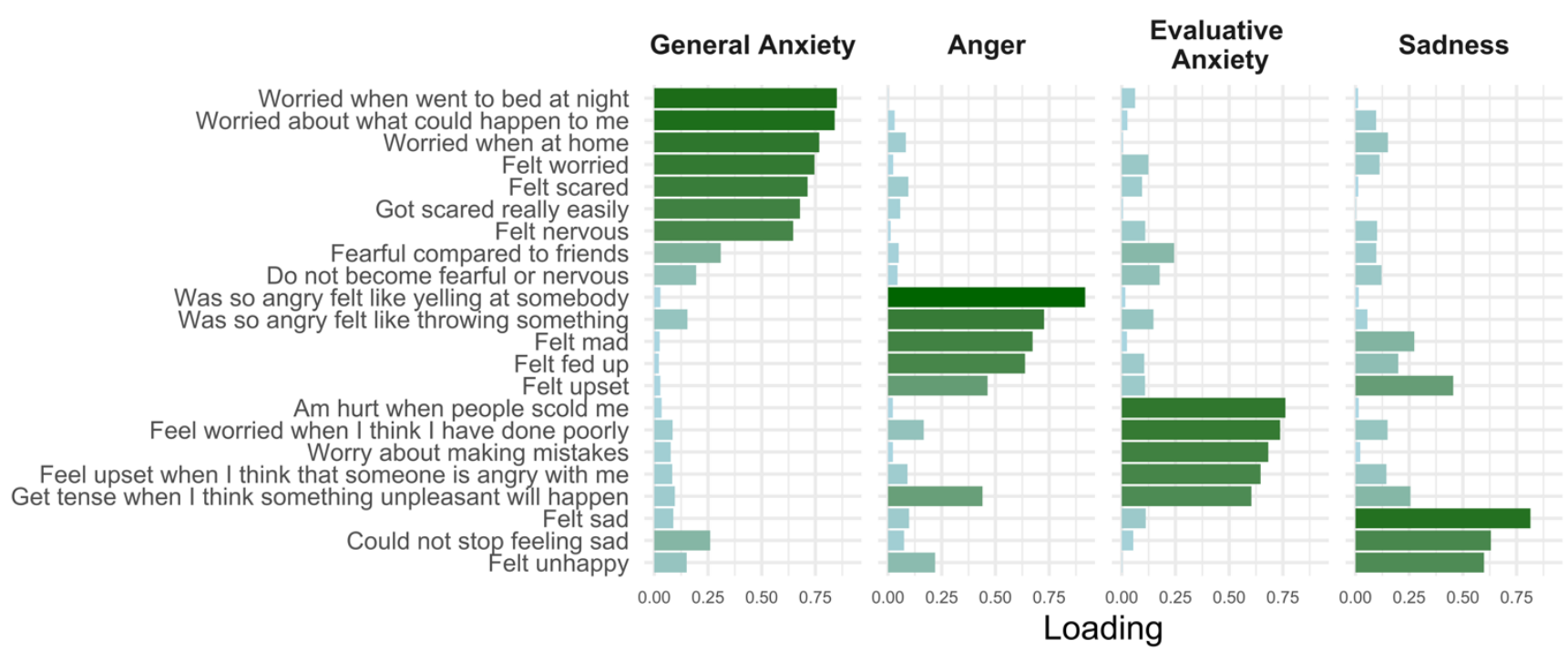


Supplementary Figure 5. EFA with all negative affect items loading plot. The absolute values of the loadings are plotted, sorted by loading strength. Higher loading strengths are depicted by larger values on the $\mathrm{x}$-axis and a darker green color.

To compare the fit of the three alternative factor solutions with the primary solution, we compared the common fit statistics used in CFA (Supplementary Table 4). With the caveat that the fit metrics for the two EFA-derived solutions may be biased upwards, we found that all metrics indicated that the primary factor solution presented in the manuscript (i.e., excluding "bisbas5" and "bisbas7") is the best fitting solution.

Supplementary Table 4. Comparison of primary factor solution and three alternative solutions.

\begin{tabular}{ccccc} 
& $\begin{array}{c}\text { Primary } \\
\text { Solution } \\
\text { (excluding } \\
\text { "bisbas5" and } \\
\text { bisbas7”) }\end{array}$ & $\begin{array}{c}\text { All Negative } \\
\text { Affect Items } \\
\text { (incl. "bisbas5" } \\
\text { and “bisbas7”) }\end{array}$ & $\begin{array}{c}\text { 3-Factor } \\
\text { Structure } \\
\text { (matching Figure } \\
\text { S5) }\end{array}$ & $\begin{array}{c}\text { 3-Factor } \\
\text { Structure } \\
\text { (general anxiety } \\
\text { and } \text { eval. anxiety } \\
\text { combined) }\end{array}$ \\
\hline CFI & .992 & .990 & .953 & .963 \\
\hline TLI & .991 & .988 & .947 & .958 \\
\hline RMSEA & $.053(.048, .059)$ & $.054(.050, .059)$ & $.128(.123, .133)$ & $.113(.109, .118)$ \\
\hline SRMR & .051 & .056 & .102 & .093 \\
\hline MFI & .792 & .741 & .255 & .343 \\
\hline Chi-squared & 523.26 & 664.34 & 2270.72 & 1813.60 \\
& $(\mathrm{df}=164, \mathrm{p}<.001)$ & $(\mathrm{df}=203, \mathrm{p}<.001)$ & $(\mathrm{df}=167, \mathrm{p}<.001)$ & $(\mathrm{df}=167, \mathrm{p}<.001)$ \\
\hline
\end{tabular}

Note: CFI: comparative fit index (should be $>=0.95)$, TLI: Tucker-Lewis index $(>.90$ indicates good fit), RMSEA: root mean square error of approximation (should be $<=0.05$, upper CI bound $<=0.10$ ), SRMR: standardized root mean square residual (should be $<=$ 0.08), MFI: McDonald fit index (higher values indicate better fit), and the chi-squared test (a non-significant $\mathrm{p}$-value suggests the model fits). 


\section{Negative affect as a summary score}

To compare our results with prior work that has studied negative affect as a singular concept, we calculated a general negative affect score and explored the relationship between overall negative affect with social functioning, life satisfaction, and age. We generated this score by conducting an EFA, using the same methods as described in the manuscript, on the twenty items as inputs. Instead of the 4-factor solution examined in the manuscript, we extracted a 1factor solution to use as a summary score of overall negative affect.

To test the extent to which overall negative affect relates to the identified social domains (emotional support, friendship, perceived hostility, loneliness, and perceived rejection) and life satisfaction, six linear regression models were conducted with overall negative affect as a predictor and each of the outcome domains as a dependent variable. The spline of age was controlled for in each model, and we accounted for multiple comparisons using the Holm method. Results showed that overall negative affect was significantly associated with emotional support, friendship, perceived hostility, loneliness, perceived rejection, and life satisfaction (Supplementary Table 5).

We then explored age-related changes in overall negative affect using the same methods described in the manuscript. A linear and spline model were tested, with overall negative affect as the dependent variable and age as the independent variable, and the model with the lowest AIC was interpreted. Results are shown in Supplementary Figure 6. A spline model (AIC = 2271.00) produced a better fitting model than a linear model ( $\mathrm{AIC}=2281.59)$. Overall negative affect showed significant non-linear changes across age $(p<.001)$, with increases appearing to be largest between the ages of 14 and 16. However, no intervals along the curve reached the 
threshold to be considered accelerated (i.e., as defined by areas where the simultaneous

confidence interval of the derivative does not include zero).

Supplementary Table 5. Results of linear models for social functioning and life satisfaction dependent variables and overall negative affect independent variable.

\begin{tabular}{lcccccc}
\hline Dependent variable & $\begin{array}{c}\text { Coeffici } \\
\text { ent }\end{array}$ & t-score & df & $\begin{array}{c}\mathrm{p}- \\
\text { value }\end{array}$ & $\begin{array}{c}\text { Corrected p- } \\
\text { value }\end{array}$ & $\begin{array}{c}\text { R-squared } \\
\text { (adjusted) }\end{array}$ \\
\hline Emotional Support & -0.29 & -8.44 & 765.86 & $<.001$ & $<.001$ & .118 \\
Friendship & -0.28 & -8.16 & 765.95 & $<.001$ & $<.001$ & .086 \\
Perceived Hostility & 0.49 & 15.70 & 766.57 & $<.001$ & $<.001$ & .261 \\
Loneliness & 0.56 & 18.86 & 766.21 & $<.001$ & $<.001$ & .328 \\
Perceived Rejection & 0.54 & 17.75 & 764.89 & $<.001$ & $<.001$ & .295 \\
Life Satisfaction & -0.38 & -9.04 & 538.87 & $<.001$ & $<.001$ & .143 \\
\hline
\end{tabular}

Note: P-values were corrected using the Holm method and were compared to an alpha level of .05 to determine significance. Spline age was controlled for in all models. Standardized regression coefficients $(\beta)$ are reported.

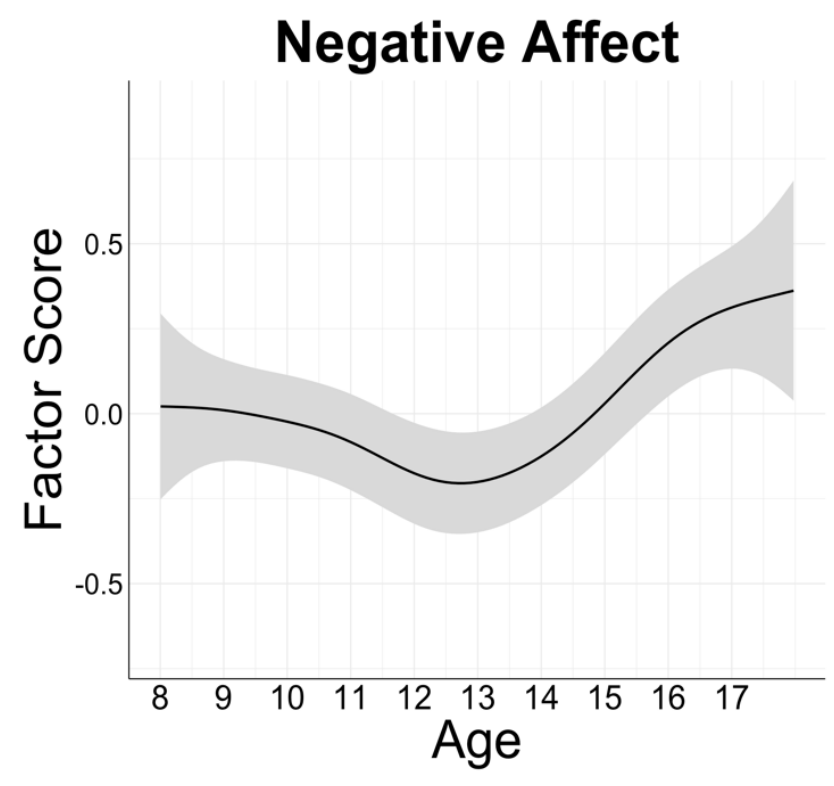

Supplementary Figure 6. Age-Related Changes in Overall Negative Affect. Solid black line depicts linear model fit. Grey shaded region depicts $95 \%$ confidence interval. Red shaded region represents the window of significant change in the GAM model. Y-axis units are factor scores. 
9. Scree plot for primary 4-factor solution

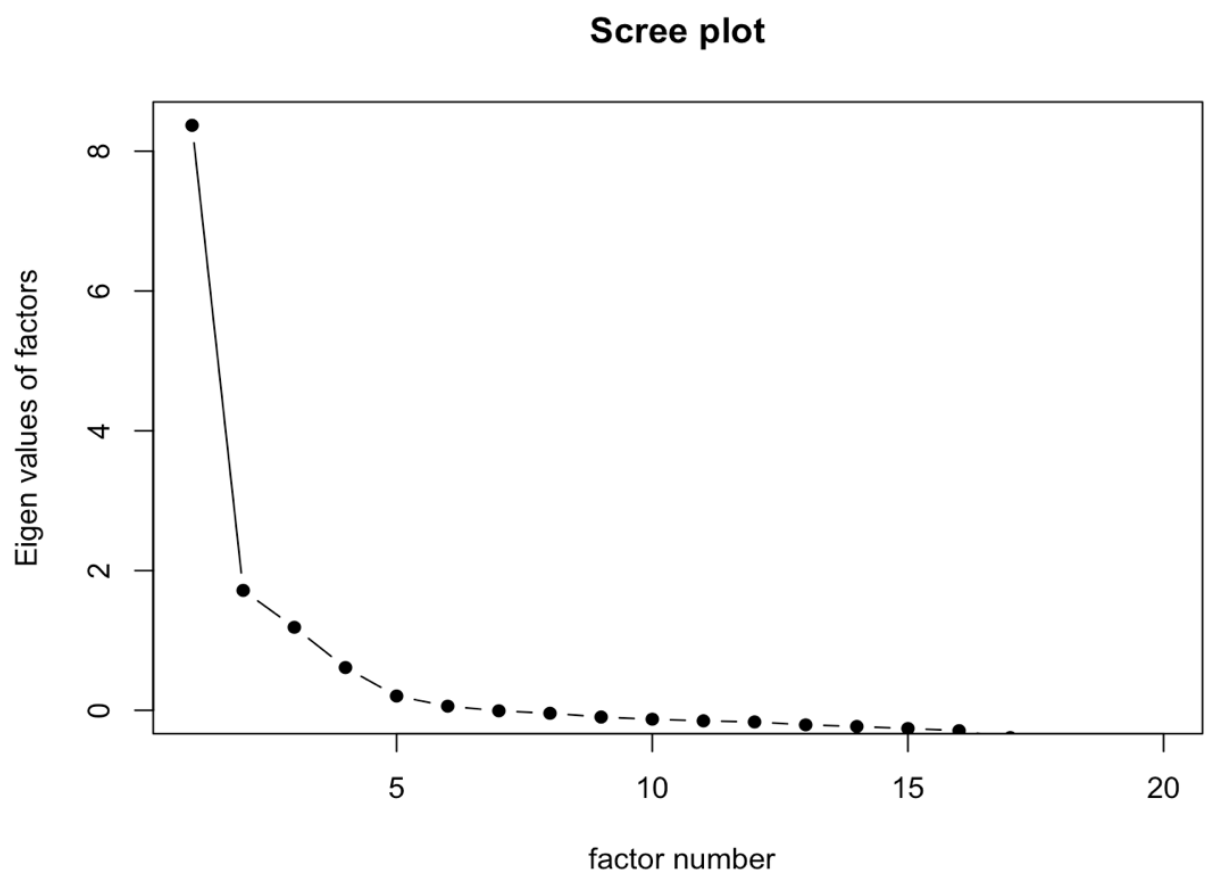

Supplementary Figure 7. Scree plot for primary 4-factor solution. Eigenvalues for one through twenty factors are plotted. 


\section{Confirmatory Factor Analysis parameter estimates}

Supplementary Table 6. Full CFA parameter estimates.

\begin{tabular}{|c|c|c|c|c|c|c|c|c|}
\hline Factor & Variable & Est & SE & $\begin{array}{l}\text { Z- } \\
\text { value }\end{array}$ & $\begin{array}{l}\text { P- } \\
\text { value }\end{array}$ & $\begin{array}{l}\text { CI } \\
\text { Lower }\end{array}$ & $\begin{array}{l}\text { CI } \\
\text { Upper }\end{array}$ & $\begin{array}{l}\text { Std. } \\
\text { Loadings }\end{array}$ \\
\hline General anxiety & PedRepAnx42 & 1.00 & 0.00 & & & 1.00 & 1.00 & 0.73 \\
\hline General anxiety & PedRepAnx43 & 1.09 & 0.04 & 30.19 & 0.00 & 1.02 & 1.16 & 0.79 \\
\hline General anxiety & PedRepAnx44 & 1.21 & 0.04 & 30.51 & 0.00 & 1.13 & 1.28 & 0.88 \\
\hline General anxiety & PedRepAnx46 & 1.10 & 0.04 & 29.25 & 0.00 & 1.03 & 1.17 & 0.80 \\
\hline General anxiety & PedRepAnx48 & 1.06 & 0.04 & 26.72 & 0.00 & 0.98 & 1.13 & 0.77 \\
\hline General anxiety & PedRepAnx50 & 1.11 & 0.04 & 27.34 & 0.00 & 1.03 & 1.19 & 0.81 \\
\hline General anxiety & PedRepAnx51 & 0.97 & 0.04 & 21.58 & 0.00 & 0.88 & 1.05 & 0.70 \\
\hline Anger & PedRepAng13 & 1.00 & 0.00 & & & 1.00 & 1.00 & 0.82 \\
\hline Anger & PedRepAng14 & 1.04 & 0.03 & 40.22 & 0.00 & 0.99 & 1.09 & 0.86 \\
\hline Anger & PedRepAng16 & 1.01 & 0.03 & 34.68 & 0.00 & 0.95 & 1.07 & 0.83 \\
\hline Anger & PedRepAng17 & 1.00 & 0.03 & 37.27 & 0.00 & 0.95 & 1.05 & 0.82 \\
\hline Sadness & PedRepAng18 & 1.00 & 0.00 & & & 1.00 & 1.00 & 0.86 \\
\hline Sadness & PedRepDep36 & 0.96 & 0.02 & 42.89 & 0.00 & 0.92 & 1.00 & 0.82 \\
\hline Sadness & PedRepDep38 & 1.02 & 0.02 & 41.92 & 0.00 & 0.97 & 1.06 & 0.87 \\
\hline Sadness & PedRepDep41 & 0.95 & 0.03 & 31.53 & 0.00 & 0.89 & 1.01 & 0.82 \\
\hline Evaluative anx. & bisbas 1 & 1.00 & 0.00 & & & 1.00 & 1.00 & 0.71 \\
\hline Evaluative anx. & bisbas 2 & 1.05 & 0.06 & 18.01 & 0.00 & 0.94 & 1.17 & 0.74 \\
\hline Evaluative anx. & bisbas 3 & 1.01 & 0.06 & 16.54 & 0.00 & 0.89 & 1.13 & 0.71 \\
\hline Evaluative anx. & bisbas6 & 0.88 & 0.06 & 14.45 & 0.00 & 0.76 & 1.00 & 0.62 \\
\hline Evaluative anx. & bisbas 4 & 1.02 & 0.06 & 16.75 & 0.00 & 0.90 & 1.13 & 0.72 \\
\hline
\end{tabular}

Note: See Supplementary Table 3 for full variable names. EST: point estimate; SE: standard error; CI Lower and CI Upper: lower and upper bounds of a 95\% confidence interval around the point estimate; Std. Loadings: standardized loadings. 
11. Age-related changes in negative affect factors (raw data points overlaid)
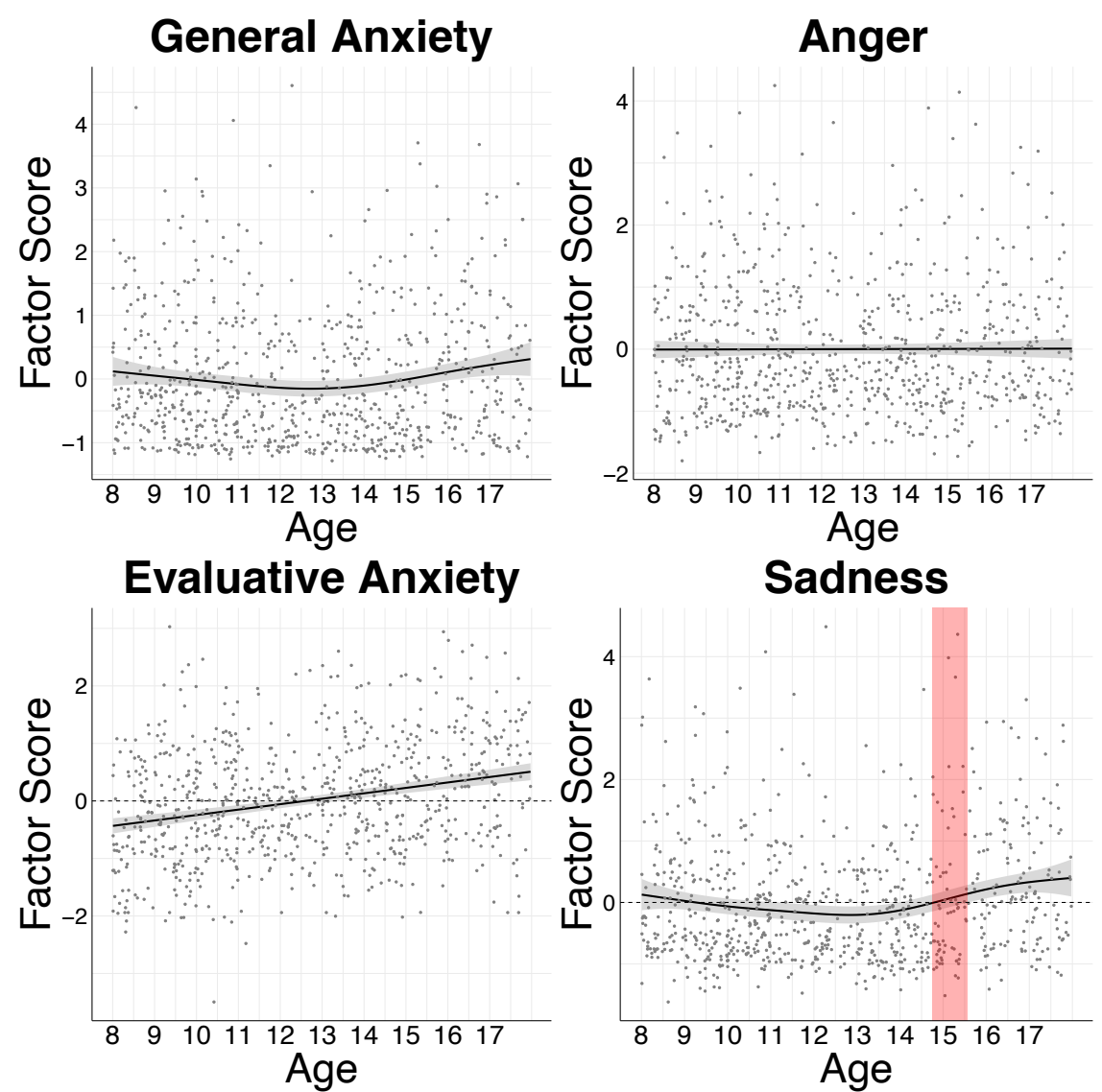

Supplementary Figure 8. Age-related changes in negative affect factors with raw data points overlaid. Solid black line depicts linear or non-linear model fit. Grey shaded region depicts $95 \%$ confidence interval. Red shaded region represents the window of accelerated change in the GAM. Y-axis units are factor scores. 


\section{Two-lines test to investigate nonlinear "u-shape" curves}

Visual inspection of the age-related changes in the negative affect (as seen in Figure 2 of the manuscript) reveals a slight "u" shape curve for two factors, general anxiety and sadness, with slight decreases in early to mid-adolescence and increases from mid to late adolescence. To explore whether this " $u$ " shape is statistically significant, we conducted a two-lines test introduced by Simonsohn (2018), which estimates an interrupted regression with two separate slopes separated by a breakpoint using the "Robin Hood" algorithm, which is shown to deliver higher power to detect u-shaped curves than other break-point-setting alternatives (Simonsohn, 2018). If the two slopes have an opposite sign and are individually statistically significant, then we reject the null hypothesis that there is no $u$-shaped effect.

We found that for both sadness and general anxiety, when the curve is broken into two regressions, there are significant decreases in early to mid-adolescence (sadness: $b=-0.06, z=-$ 2.07, $p=.038$, general anxiety: $b=-0.08, z=-2.51, p=.012$ ) followed by significant increases in mid to late adolescence (sadness: $b=0.17, z=4.09, p<.001$, general anxiety: $b=0.12, z=$ $2.76, p=.006)$ (Supplementary Figure 9). The breakpoint, or the age at which the factor scores are at a minimum, is around 13 years of age for both factors (sadness: 13.3 years, general anxiety: 13.2). As this analysis is an exploratory post-hoc analysis, we did not correct for multiple comparisons. 

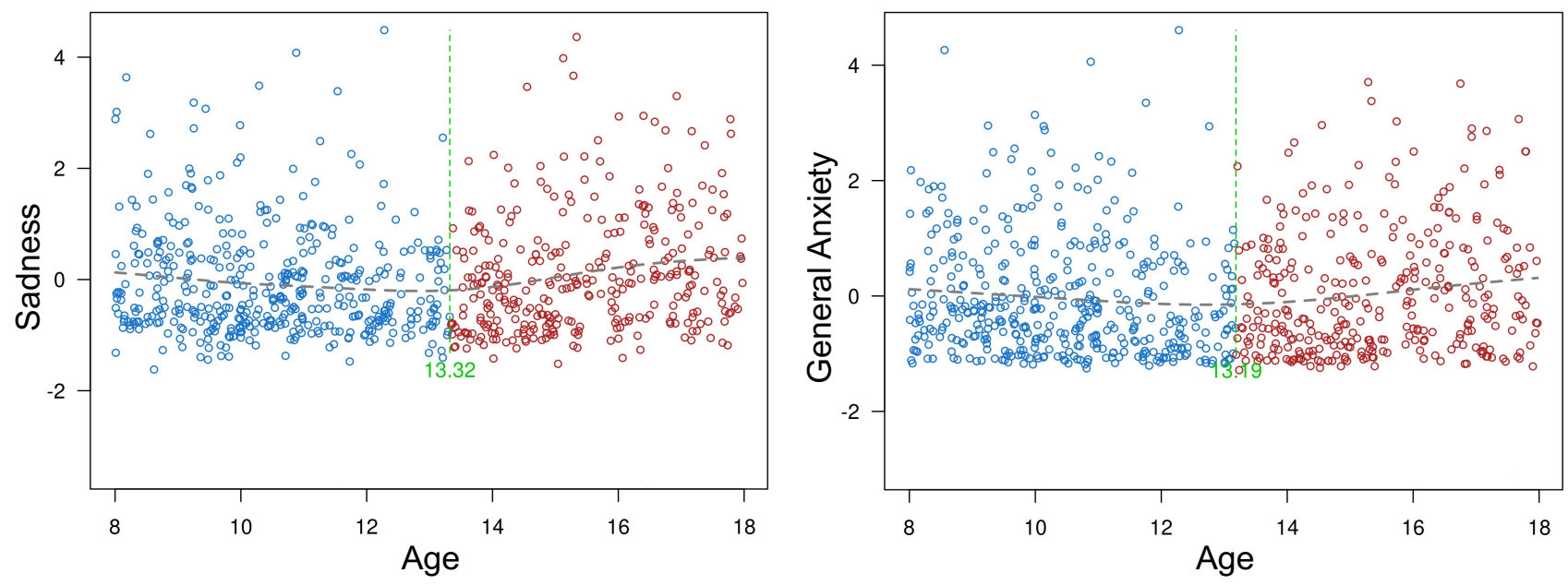

Supplementary Figure 9. Simonsohn's two-lines procedure. Blue points and text indicate the first line in the interrupted regression; red points and text indicates the second interrupted regression. The green dotted line and text indicates the breakpoint, or lowest point on the " $u$ " shape separating the two regressions. 


\section{Multi-group CFA to identify whether the factor structure is invariant across gender and age bins}

We explored whether the factor structure identified was invariant across gender and age in our sample. It was not possible to evaluate the invariance of the factor structure treating age as a continuous variable, so age was treated according to three age groupings ( 8 to 11 years, 12 to 14, and 15 to 17 years). For gender, we compared males and females, and for age we compared the three age bins. We conducted a multi-group CFA using the lavaan package in $\mathrm{R}$ to assess invariance. We compared the fit of three different models to the data: 1) configural invariance, where the same factor structure is evident in both/all groups, 2) weak invariance, where the factor loadings are constrained to be equal across groups, and 3) strong invariance, where the factor loadings and intercepts (i.e., each item's origin value, similar to the concept of a yintercept in a regression context) are equal across groups. These tests are typically conducted sequentially and once a test indicates non-invariance no further tests are run. A poor multi-group model fit at the configural invariance stage would suggest that the overall factor structure does not hold up for all groups. If constraining the factor loadings in the weak invariance stage results in a poorer model fit, it suggests the factor loadings are not similar across groups. If constraining both the factor loadings and intercepts result in a poorer model fit, it suggests the item intercepts are not similar across groups.

We identify a "poorer model fit" using the Comparative Fit Index (CFI) criterion, which is a widely used and empirically supported criterion (Chen, 2007; Cheung \& Rensvold, 2002) that defines invariance as a $<.01$ change in CFI between models. The $\Delta$ CFI criterion avoids demonstrated problems with using chi-square values to determine invariance, as it is independent of model complexity and sample size (Cheung \& Rensvold, 2002). We also report the chi square test statistic, root mean square error of approximation, standardized root mean square residual, 
and McDonald fit index to fully describe the model fits, but did not use these values to determine invariance. The three models for both gender and age, including the "overall model" (i.e., the original CFA model using the whole sample not split into groups), are compared in

\section{Supplementary Table 7.}

For gender, the configural invariance model did not differ significantly from the weak invariance model $(\mathrm{CFI}$ diff $=.001)$ and the strong invariance model did not differ significantly from the weak invariance model $(\mathrm{CFI}$ diff $<.001)$, providing support for both weak and strong invariance in our factor structure between males and females. For age, the configural invariance model did not differ significantly from the weak invariance model (CFI diff $=.002)$, and the strong invariance model did differ significantly from the weak invariance model (CFI diff $=$ $.001)$. We therefore conclude that both weak and strong invariance are supported in our dataset across the three age bins examined. These analyses demonstrate that the factor structure is consistent across males and females, and across the three age groups tested.

Supplementary Table 7. Multi-group CFA fit statistics.

\begin{tabular}{llllrrrr}
\hline Group & Model & $\chi^{2}$ & df & \multicolumn{2}{c}{ CFI } & RMSEA & \multicolumn{2}{l}{ SRMR } & \multicolumn{2}{l}{ MFI } \\
\hline \multirow{3}{*}{ Gender } & Overall Model & 523.257 & 164 & 0.992 & 0.053 & 0.051 & 0.792 \\
\hline \multirow{4}{*}{ Age } & Configural Invariance & 669.785 & 328 & 0.993 & 0.052 & 0.058 & 0.801 \\
& Weak Invariance & 728.755 & 344 & 0.992 & 0.054 & 0.060 & 0.778 \\
& Strong Invariance & 763.946 & 395 & 0.992 & 0.049 & 0.058 & 0.786 \\
\hline & Configural Invariance & 756.549 & 492 & 0.995 & 0.046 & 0.061 & 0.842 \\
& Weak Invariance & 851.651 & 524 & 0.993 & 0.049 & 0.066 & 0.808 \\
& Strong Invariance & 999.048 & 626 & 0.992 & 0.048 & 0.063 & 0.784 \\
\hline
\end{tabular}

$\chi^{2}$ : chi-squared test, df: degrees of freedom, CFI: comparative fit index, RMSEA: root mean square error of approximation, SRMR: standardized root mean square residual, MFI: McDonald fit index. 


\section{Testing gender as a moderator between negative affect factors and functioning out- comes}

We also explored whether gender moderated the observed relationships between the negative affect factors and the social functioning and life satisfaction outcomes. For each significant relationship reported in Table 1 of the manuscript, we tested whether the interaction between gender and the negative affect factor was significantly related to the outcome variable (Supplementary Table 8). We found that after correcting for multiple comparisons, gender did not significantly moderate any of the negative affect - functioning outcome relationships. Before pvalue correction, we observed a significant moderation by gender on the relationship between anger and perceived hostility (such that males show stronger relationship than females) as well as between evaluative anxiety and loneliness (such that females show a stronger relationship than males) (Supplementary Figure 10). These potential gender differences highlight an important area for future study.
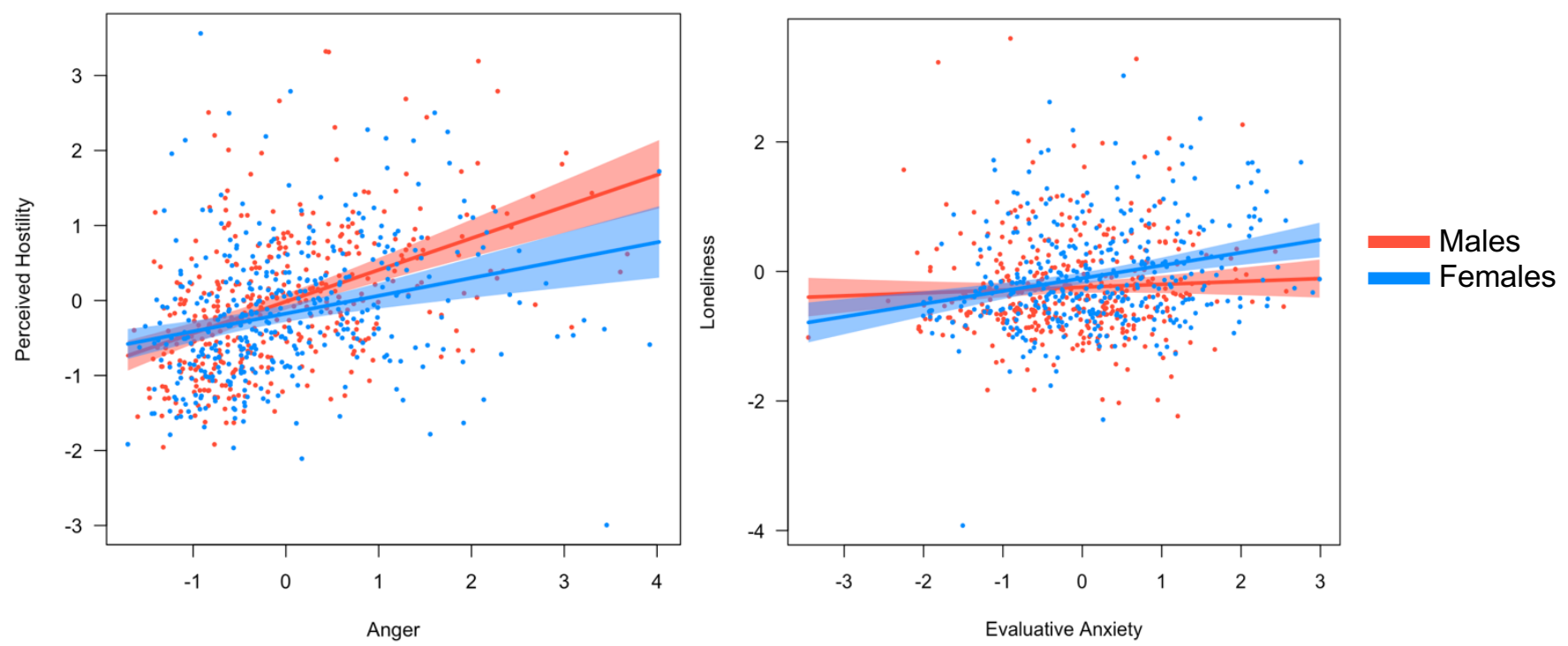

Supplementary Figure 10. Effect of gender and negative affect on social functioning. The relationship between anger and perceived hostility split by gender (left) and the relationship between evaluative anxiety and loneliness split by gender (right) are plotted. X-axis units are factor scores, $y$-axis units are scaled total scores. Solid red and blue lines depict linear model fits for 
males and females (respectively); red and blue shaded regions depict 95\% confidence interval for males and females (respectively).

Supplementary Table 8. Gender as a moderator. Testing whether gender moderates the significant relationships between negative affect factors and functioning outcome measures.

\begin{tabular}{|c|c|c|c|c|c|c|}
\hline Dependent variable & Independent variables & Coefficient & $\begin{array}{c}\text { t- } \\
\text { score }\end{array}$ & $\mathrm{df}$ & $\begin{array}{c}\text { p- } \\
\text { value }\end{array}$ & $\begin{array}{c}\text { Corrected } \\
\text { p-value }\end{array}$ \\
\hline \multirow[t]{2}{*}{ Emotional Support } & General Anxiety*Gender & 0.11 & 1.10 & 753.96 & .270 & 1.000 \\
\hline & Sadness*Gender & -0.002 & -0.02 & 753.96 & .984 & 1.000 \\
\hline Friendship & Sadness*Gender & -0.12 & -1.37 & 758.10 & .172 & 1.000 \\
\hline \multirow[t]{2}{*}{ Perceived Hostility } & Anger*Gender & -0.18 & -2.37 & 758.10 & .018 & .182 \\
\hline & Sadness*Gender & 0.11 & 1.34 & 758.10 & .181 & 1.000 \\
\hline \multirow[t]{2}{*}{ Loneliness } & Eval Anxiety*Gender & 0.15 & 2.51 & 758.75 & .012 & .134 \\
\hline & Sadness*Gender & -0.01 & -0.14 & 758.75 & .889 & 1.000 \\
\hline \multirow[t]{2}{*}{ Perceived Rejection } & Anger*Gender & -0.15 & -1.96 & 756.34 & .051 & .455 \\
\hline & Sadness*Gender & -0.09 & -1.18 & 756.34 & .238 & 1.000 \\
\hline \multirow[t]{2}{*}{ Life Satisfaction } & Anger*Gender & -0.11 & -1.27 & 530.63 & .205 & 1.000 \\
\hline & Sadness*Gender & -0.16 & -1.54 & 530.63 & .124 & .994 \\
\hline \multicolumn{7}{|c|}{$\begin{array}{l}\text { Note: P-values were corrected using the Holm method and were compared to an alpha level of } .05 \text { to } \\
\text { determine significance. Bold text indicates significant models. Spline age was controlled for in all } \\
\text { models. Gender is coded as male }=1, \text { female }=2 \text {. }\end{array}$} \\
\hline
\end{tabular}




\section{Negative affect changes as a function of pubertal development}

As research suggests that pubertal development may relate to differences in negative affect during adolescence (e.g., Forbes et al., 2004), we used a model comparison approach to explore how the different forms of negative affect identified vary across age versus pubertal stage, as measured by the Pubertal Development Scale (PDS, Petersen et al., 1988). For the youngest participants (age 8), parent-reported PDS data were collected and are reported; for older participants (ages 14-17), participant-reported data were collected and are reported, and for participants ages 9-13, both parent and participant-reported data were collected, thus an average of both scores was calculated and used for the current analysis. For each negative affect factor, a model with age was compared to a model with puberty based on AIC values, matching the methods detailed in the manuscript (Supplementary Table 9).

We found that for general anxiety, age is the better fitting model; for anger, neither age nor puberty are significant predictors; for evaluative anxiety, both age and puberty are significant predictors, with puberty as the better fitting model (Supplementary Figure 11); and for sadness, both age and puberty are significant predictors, with age as the better fitting model.

Supplementary Table 9. Comparing changes in different forms of negative affect across age versus pubertal development.

\begin{tabular}{llccc}
\hline Dependent variable & Model & AIC & p-value & Corrected p-value \\
\hline General Anxiety & Age & $\mathbf{2 2 4 6 . 2 5}$ & $\mathbf{. 0 1 1}$ & $\mathbf{. 0 4 4}$ \\
& Puberty & 2252.03 & .056 & .168 \\
\multirow{5}{*}{ Anger } & Age & 2274.06 & .913 & .913 \\
& Puberty & 2272.54 & .217 & .434 \\
Evaluative Anxiety & Age & 2154.02 & $<.001$ & $<.001$ \\
& Puberty & $\mathbf{2 1 2 7 . 6 0}$ & $<.001$ & $<.001$ \\
\multirow{2}{*}{ Sadness } & & & & \\
& Age & $\mathbf{2 1 9 3 . 3 6}$ & $<.001$ & $\mathbf{. 0 0 1}$ \\
& Puberty & 2201.68 & .002 & .010 \\
\hline
\end{tabular}


Note: Bold text indicates best fitting model for each dependent variable, as determined by AIC. AIC $=$ Akaike Information Criterion. P-value refers to approximate significance of smooth age terms. P-values were corrected using the Holm method and were compared to an alpha level of .05 to determine significance.
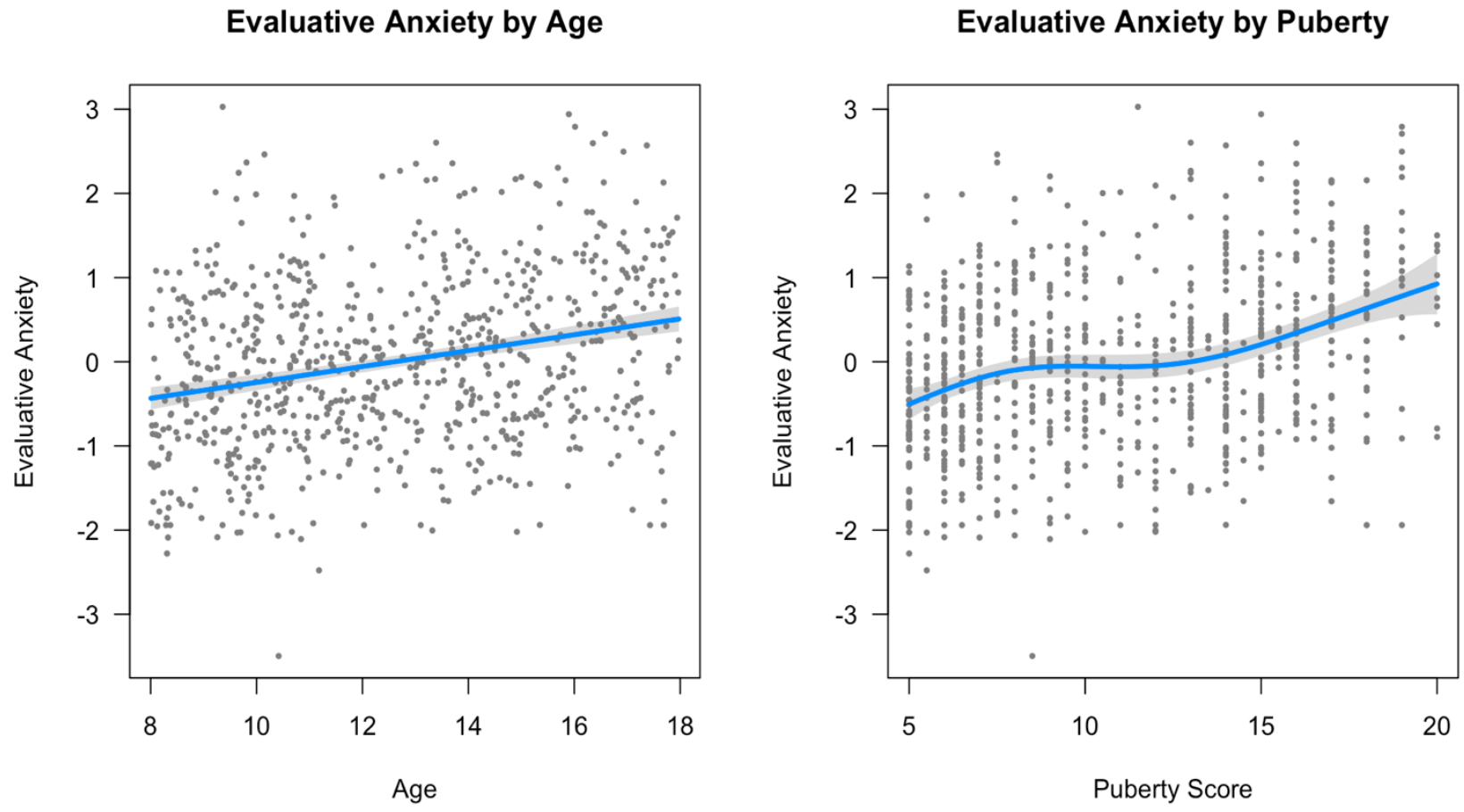

Supplementary Figure 11. Age and puberty-related changes in evaluative anxiety. Solid blue line depicts linear or non-linear model fit. Grey shaded region depicts 95\% confidence interval. 


\section{Age-related changes in the social functioning and life satisfaction outcome domains}

To provide a comprehensive exploration of the age-related changes in our sample, we conducted a supplementary analysis using the same generalized additive modeling approach described in the manuscript to identify age-related changes in each of the social functioning domains and life satisfaction. Six regressions were run, each with a functioning outcome as the dependent variable and the spline of age as the independent variable. Emotional support $(p<.001)$, perceived hostility $(p<.001)$, and loneliness $(p=.003)$ showed significant non-linear age-related changes; friendship $(p=.126)$, perceived rejection $(p=.139)$, and life satisfaction $(p=.126)$ did not show significant age-related changes (Supplementary Figure 12). P-values were corrected using the Holm method.
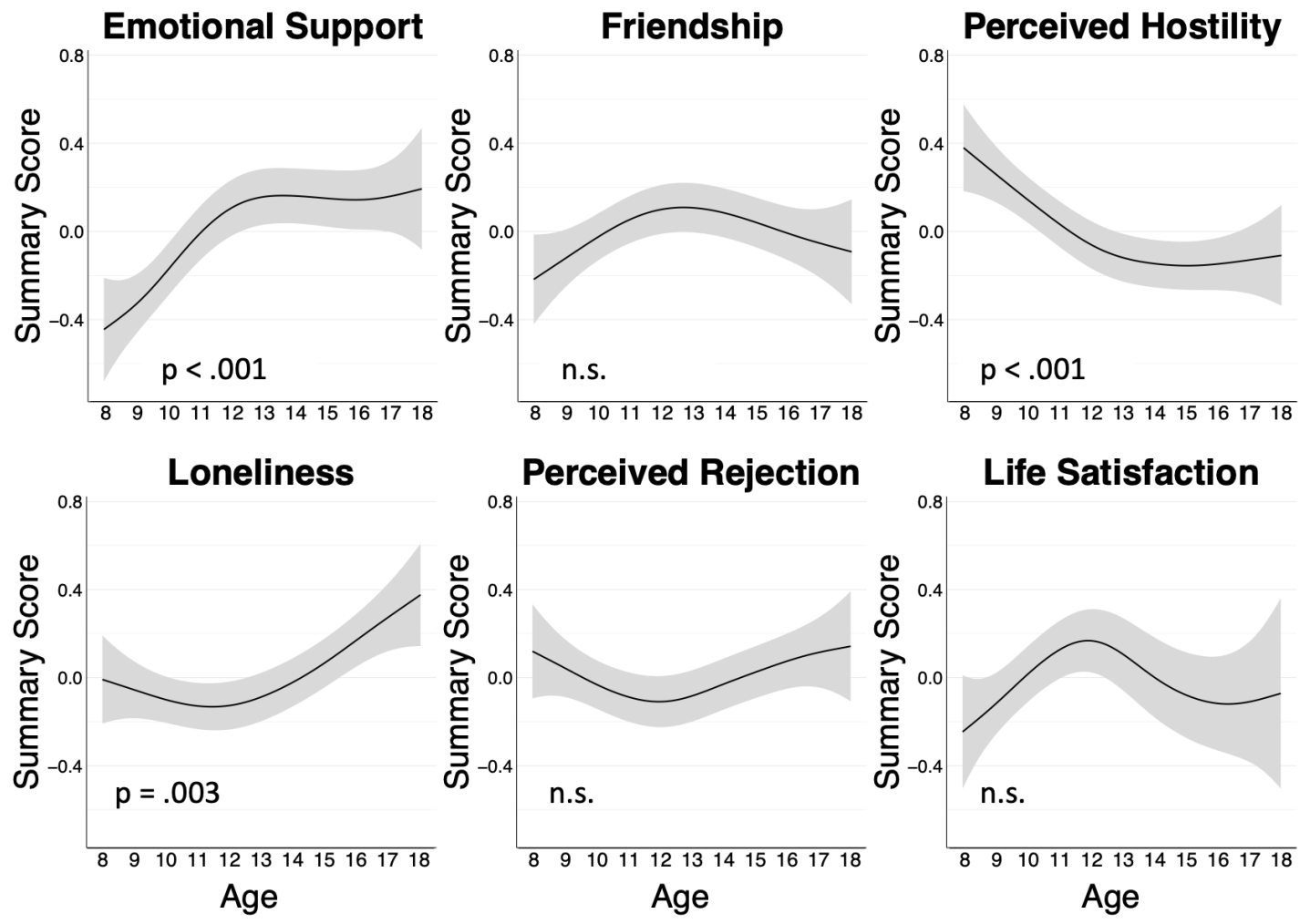

Supplementary Figure 12. Age-related changes in social functioning and life satisfaction outcome domains. Solid black line depicts model fit. Grey shaded region depicts $95 \%$ confidence interval. 


\section{Exploration of the relationship between negative affect factors and clinically rele- vant measures}

Our primary analyses focus on normative experiences of affect in a healthy

developmental sample. However, given the link between negative affect and psychopathology and increases in the onset of psychopathology during the age range of our sample, we conducted a supplementary analysis to examine the degree to which different facets of negative affect predict depressive, anxiety, and somatic problems. We examined six DSM-oriented scales that map broadly onto DSM diagnostic categories from the Child Behavior Checklist (CBCL; Achenbach, 1997). Regressions were conducted as described in the manuscript; for each CBCL DSM-oriented sub-scale score, a separate regression model was run to identify which negative affect factors (all included as linear predictors) significantly predict that sub-scale score (Supplementary Table 10).

We found that the general anxiety and evaluative anxiety factor scores were significantly and positively associated with the DSM-oriented anxiety problems scale and the anger factor score was significantly and positively associated with the DSM-oriented oppositional defiant problems and conduct problems scales. These findings show that in addition to the negative affect factors showing distinct relationships with normative differences in social functioning and life satisfaction, they also show relationships with clinically oriented outcomes with some specificity (i.e., affective anxiety related to clinical anxiety). Future research is needed to further investigate the relationship between affective experiences in a healthy adolescent sample with symptoms of psychopathology. 
Supplementary Table 10. Results of linear models for CBCL DSM-oriented sub-scales.

\begin{tabular}{|c|c|c|c|c|c|c|}
\hline Dependent variable & $\begin{array}{l}\text { Independent } \\
\text { variables }\end{array}$ & Coefficient & $\mathrm{t}$-score & $\mathrm{df}$ & $\mathrm{p}$-value & $\begin{array}{c}\text { Corrected p- } \\
\text { value }\end{array}$ \\
\hline Depressive & General Anxiety & 0.07 & 1.38 & 764.00 & .169 & 1.000 \\
\hline \multirow[t]{3}{*}{ Problems } & Anger & 0.06 & 1.35 & 764.00 & .177 & 1.000 \\
\hline & Evaluative Anxiety & 0.09 & 2.42 & 764.00 & .016 & .255 \\
\hline & Sadness & 0.12 & 2.68 & 764.00 & .008 & .144 \\
\hline \multirow[t]{4}{*}{ Anxiety Problems } & General Anxiety & 0.28 & 5.46 & 763.98 & $<.001$ & $<.001$ \\
\hline & Anger & -0.03 & -0.58 & 763.98 & .560 & 1.000 \\
\hline & Evaluative Anxiety & 0.12 & 3.30 & 763.98 & .001 & .021 \\
\hline & Sadness & 0.01 & 0.13 & 763.98 & .894 & 1.000 \\
\hline \multirow[t]{4}{*}{ Somatic Problems } & General Anxiety & 0.10 & 1.80 & 764.00 & .072 & 1.000 \\
\hline & Anger & 0.11 & 2.52 & 764.00 & .012 & .204 \\
\hline & Evaluative Anxiety & -0.06 & -1.57 & 764.00 & .116 & 1.000 \\
\hline & Sadness & 0.05 & 1.00 & 764.00 & .320 & 1.000 \\
\hline Attention Deficit / & General Anxiety & 0.06 & 1.16 & 764.00 & .246 & 1.000 \\
\hline Hyperactivity & Anger & 0.12 & 2.63 & 764.00 & .009 & .156 \\
\hline \multirow[t]{2}{*}{ Problems } & Evaluative Anxiety & -0.05 & -1.33 & 764.00 & .185 & 1.000 \\
\hline & Sadness & 0.02 & 0.44 & 764.00 & .664 & 1.000 \\
\hline Oppositional & General Anxiety & -0.02 & -0.38 & 764.00 & .704 & 1.000 \\
\hline \multirow[t]{3}{*}{ Defiant Problems } & Anger & 0.21 & 4.53 & 764.00 & $<.001$ & $<.001$ \\
\hline & Evaluative Anxiety & -0.07 & -1.68 & 764.00 & .093 & 1.000 \\
\hline & Sadness & $-0.0^{`}$ & -0.12 & 764.00 & .908 & 1.000 \\
\hline \multirow[t]{4}{*}{ Conduct Problems } & General Anxiety & -0.03 & -0.64 & 758.04 & .524 & 1.000 \\
\hline & Anger & 0.18 & 4.06 & 758.04 & $<.001$ & .001 \\
\hline & Evaluative Anxiety & -0.12 & -3.01 & 758.04 & .003 & .054 \\
\hline & Sadness & 0.03 & 0.75 & 758.04 & .454 & 1.000 \\
\hline
\end{tabular}

Note: P-values were corrected using the Holm method (6 total regressions each with 4 affect factors $=$ 24 total tests) and were compared to an alpha level of .05 to determine significance. Bold text indicates significant models. Spline age was controlled for in all models. 